\title{
Multi-attribute group decision making based on sine trigonometric spherical fuzzy aggregation operators
}

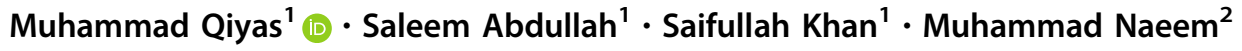

Received: 2 December 2020 / Accepted: 24 January 2021 / Published online: 22 March 2021

(C) The Author(s), under exclusive licence to Springer Nature Switzerland AG part of Springer Nature 2021

\begin{abstract}
Spherical fuzzy set (SFS) is also one of the fundamental concepts for address more uncertainties in decision problems than the existing structures of fuzzy sets, and thus its implementation was more substantial. The well-known sine trigonometric function maintains the periodicity and symmetry of the origin in nature and thus satisfies the expectations of the experts over the multi parameters. Taking this feature and the significance of the SFSs into the consideration, the main objective of the article is to describe some reliable sine trigonometric laws for SFSs. Associated with these laws, we develop new average and geometric aggregation operators to aggregate the Spherical fuzzy numbers. Then, we presented a group decision-making strategy to address the multi-attribute group decision-making problem using the developed aggregation operators. To verify the value of the defined operators, a MAGDM strategy is provided along with an application for the selection of an authentic COVID-19 laboratory. Moreover, a comparative study is also performed to present the effectiveness of the developed approach.
\end{abstract}

Keywords Spherical fuzzy sets - Sine trigonometric spherical fuzzy aggregation operators - Decision making

\section{Introduction}

Multi-attribute group decision-making (MAGDM) method is one of the most relevant and evolving topics explaining how to choose the finest alternative with a community of experts with some attributes. There are two relevant tasks in this system. The first is to define the context in which the values of the various parameters are effectively calculated, while the second is to summarize the defined information. Generally, the information describing the objects is taken

Muhammad Qiyas

muhammadqiyas@awkum.edu.pk

Saleem Abdullah

saleemabdullah@awkum.edu.pk

Saifullah Khan

saifullah.wazir@awkum.edu.pk

Muhammad Naeem

naeemtazkeer@yahoo.com

1 Department of Mathematics, Abdul Wali Khan University, Mardan, Pakistan

2 Department of Preparatory Year, Umm Al-Qura University, Makkah, Saudi Arabia mostly in the form of deterministic or crisp in nature. With the increasing complexity of a system on a daily basis, however, it is difficult to aggregate the data, from the logbook, resources and experts, in the crisp form. Therefore, Zadeh (1965) developed the core concept of fuzzy set (FS), and also Atanassov (1986) work on it and further develop a new idea of intuitionistic fuzzy set (IFS). Chen and Chang (2016a) proposed a fuzzy multi-attribute decision making based on transformation techniques of intuitionistic fuzzy values and intuitionistic fuzzy geometric averaging operators.

Chen et al. (2016b) developed a multi-criteria decision making based on the TOPSIS method and similarity measures between intuitionistic fuzzy values. Chen et al. (2016c) defined a novel similarity measure between intuitionistic fuzzy sets based on the centroid points of transformed fuzzy numbers with applications to pattern recognition. Chen et al. (2016d) introduced fuzzy multiattribute group decision making based on intuitionistic fuzzy sets and evidential reasoning methodology. Zeng et al. (2019a) developed a multi-attribute decision making based on novel score function of intuitionistic fuzzy values and modified VIKOR method. Yager (2013) developed the 
Pythagorean fuzzy sets (PyFSs), Torra (2010) was defined the idea of a hesitant fuzzy sets, which are used by scholars to communicate the information clearly. In IFS, it is observed that each object has two membership grades, positive $\mu$ and the negative $v$, which satisfying the condition $0 \leq \mu+v \leq 1$, and for all $\mu, v$ are lying in closed interval 0 and 1 . However, in the Pythagorean fuzzy sets, this constraint is relaxed from $\mu+v \leq 1$, to $\mu^{2}+v^{2} \leq 1$ for $\mu, v \in[0,1]$. Using this concept, many researchers have strongly addressed the define two critical tasks and discretion the techniques under the different aspects. The basic results of IFSs and Pythagorean fuzzy sets, such as operational laws De et al. (2000) and Gou et al. (2016), exponential operational laws Gou and Xu (2017), distance or similarity measures (Garg and Kumar 2018a; Hwang et al. 2018), and information entropy Grag (2019a). Many researchers (Xu and Yager 2006; Xu 2007; Garg 2016a, 2017a; Liu 2013; Liu et al. 2019), under IFS, defined some basic aggregation operators $(A O S)$, like as average and geometric, interactive AOs, Hamacher AOs. While for Pythagorean fuzzy sets, some basic operators are proposed by Peng and Yang (2015). To solve the MAGDM problems, Garg (2016b, 2017b), presented some basic concept of Einstein aggregation operators. Some extended aggregation operators dependent on the intuitionistic and Pythagorean fuzzy information, including the TOPSIS technique based on intuitionistic fuzzy Garg and Kumar (2018a) and Pythagorean fuzzy Zhang and Xu (2014); partitioned Bonferroni mean Nie et al. (2019); Maclaurin symmetric mean (Qin and Liu 2014; Gao 2018a). Apart from this, Yager (2016), intuitively developed the idea of q-rung orthopair fuzzy sets (q-ROFSs). Gao et al. (2018a), developed the basic idea of the continuities and differential of q-ROFSs. Peng et al. (2018) presented exponential , logarithmic operation laws for q-ROFNs. Liu and Wang (2018b) developed weighted average and geometric aggregation operators for q-ROFNs. Liu and Wang (2018a) developed a MAGDM method based on Archimedean Bonferroni Operators of q-rung orthopair fuzzy numbers. Liu et al. (2018) defined a MAGD method based on q-rung orthopair fuzzy Heronian mean operators. Liu and Liu (2019a) proposed a MAGDM method based on power Bonferroni operators of linguistic q-rung orthopair fuzzy numbers. Liu and Liu (2019b) introduced a MAGDM method of linguistic q-rung orthopair fuzzy power Muirhead mean operators based on entropy weight. Wei et al. (2019), defined the idea of some q-ROF Maclaurin symmetric mean operators. Wang et al. (2019c), introduced similarity measure of q-ROFSs. Liu and Wang (2020) defined a MADM method based on q-rung orthopair fuzzy generalized Maclaurin symmetric mean operators.
While, the idea of IFSs and Pythagorean FSs are widely studied and implemented in various fields. But their ability to express the information is still limited. Thus, it was still difficult for the experts and their corresponding information to convey the information in such sets. To overcome this information, the notion of the picture fuzzy sets (PFSs), which is defined by Cuong and Kreinovich (2013). Thus, it was clearly noticed that PFS is the extended form of the IFSs, to accommodate some more ambiguities. In picture fuzzy sets, each object observed by defining three grades of the member named as membership $\mu$, neutral $\eta$ and non-membership $v$ with constraint that $\mu+\eta+v \leq 1$, for $\mu, \eta, v \in[0,1]$. The definition of the PFS will convey the opinions of experts like "yes" "abstain" "no" and "refusal" while avoiding missing evaluation details and encouraging the reliability of the acquired data with the actual environment for decision-making. Although the concept of PFSs is widely studied and applied in different fields. And their extension focus on the basic operational laws, which is the important aspect of the PFS as well as aggregation operators $(A O s)$, which are an effective tool by the help of these AOs, we obtain raking of the alternatives by providing the comprehensive values to the alternatives. Wei (2017), developed some operations of the PFS. Son (2017), developed measuring analogousness in PFSs . Apart from these, several other kinds of the AOs of the PFSs have been developed such as logarithmic PF aggregation operators, which are presented by Khan (2019), Wang et. al. (2018) presented PF normalized projectionbased VIKOR method, Wang et al. (2019a), develop PF Muirhead mean operators. Wei et al. (2019), developed Bidirectional projection method for PFSs. Ashraf et al. (2018, 2019a) and Zeng et al. (2019b) developed the idea of different approaches to MAGDM problems, picture fuzzy linguistic sets and exponential Jensen PF divergence measure, respectively. Khan (2019), presented PF aggregation based on Einstein operation. Qiyas et al. (2019), presented linguistic PF Dombi aggregation operators. Cuong and Kreinovich (2013) defined some operations and defined some picture fuzzy logic operators for fuzzy derivation forms. The properties of PF t-norm \& conorm are examined by Cuong et al. (2016). Phong et al. (2014) analyze some design of PF relations. Akram et al. (2020) proposed a decision-making model under complex PF Hamacher AOs. Ahmad et al. (2019) defined new operations on interval-valued picture fuzzy set, interval-valued picture fuzzy soft set and their applications. Garg (2017c) developed some picture fuzzy aggregation operators and an approach for multi-criteria decision making. Lin et al. (2020) proposed a novel picture fuzzy MCDM model based on the extended MULTIMOORA method to solve the site selection of the car sharing station. Liu et al. (2019) defined the similarity measures for interval-valued picture 
fuzzy sets and discussed their applications in decision making. Recently, Khan et al. (2019) defined the new concept about logarithmic operation laws for PFSs.

To address this limitation which PFN cannot handle, Shahzaib et al. (2019b) defined the notion of the Spherical fuzzy set (SFS) for the first time and identified some aggregation operators with the Spherical fuzzy information problem for MADM. In the SFS, all the membership degrees are gratifying the condition $0 \leq\left(\mu_{I}(r)\right)^{2}+\left(\eta_{I}(r)\right)^{2}+\left(v_{I}(r)\right)^{2} \leq 1 \quad$ rather than $0 \leq \mu_{I}(r)+\eta_{I}(r)+v_{I}(r) \leq 1$ as in PFSs. Gundogdu et al. (2019) specified the TOPSIS method for SFS and give an example of multi-attribute decision making problem. Huanhuan et al. (2019) defined SLFS, which combines the concept of LFS with SFS. Ashraf et al. (2019c, 2019d) using the Dombi method, described some SF aggregation operators and discussed their decision-making application, also studied the presentation of SF t-norm and conorm. Jin et al. (2019) developed some Spherical fuzzy logarithmic AOs based on entropy and their application in decision support systems. Jin et al. (2019) proposed the linguistic Spherical fuzzy AOs and discussed their applications in MADM problems. Rafiq et al. (2019) introduced some cosine similarity measures of Spherical fuzzy sets and their applications in decision making. Zeng et al. (2019c) developed a Covering-based Spherical fuzzy rough set model hybrid with TOPSIS for MADM. Mahmood et al. (2019) define a model for decision making and medical diagnosis problems using the concept of SFSs.

Among the above aspects, it is very clear that operational laws play main role model for any aggregation process. Besides these mathematical logarithmic functions another important feature is the sine trigonometry feature, which plays a main role during the fusion of the information. In this way, taking into consideration the advantages and usefulness of the sine trigonometric function, some new sine trigonometric operational laws need to be developed for SFSs and their behavior studied. Consequently, the paper's purpose is to develop some new operation laws for SFSs and also give the MAGDM algorithm for managing the information for SFSs evaluation. Describe several more sophisticated operational laws for SFSs as well as a novel entropy to remove the weight of the attributes to prevent subjective, objective aspects. Some more generalized functional aggregation operators are presented with help of the defined sine trigonometric operational laws (STOLs) for spherical fuzzy numbers (SFNs), many basic relations between the developed AOs are discussed and give a novel MAGDM technique depending on the developed operators to solve the group decision-making problems. And finally, the proposed approach compared with the existing method. Therefore, the goals and the motivations of this paper are as follows:

(1) To present some more advanced operational laws for SFSs by combining the features of the ST and SFNs.

(2) A novel entropy is presented to extract the attributes' weight for avoiding the influence of subjective and objective aspects.

(3) To present some more generalized functional AOs with the help of the defined STOLs for SFNs. Also, the several fundamental relations between the proposed AOs are derived to show its significance.

(4) To present a novel MAGDM method based on the proposed operators to solve the group decisionmaking problems. The consistency of the proposed method is confirmed through these examples, and their evaluations are carried out in detail.

In the second Section of the article, we can define some related to SFS. In Section 3, we define the new SFS operational laws based on sine trigonometric function and their properties. In Section 4, we presented a series of AOs along with their required properties, based on sine trigonometric operational laws. Section 5, provides the basic connection between the developed AOs. In Section 6, using the new aggregation operators, we introduce a new MAGDM approach and give detailed steps. An example in the field of medical line using the SFNs information is given in Section 7, and to validate the new method and comparative study is carried out with the current methods are also given. Finally, the work is concluded in Section 8.

\section{Preliminaries}

Some fundamental ideas about the Spherical fuzzy set (SFS) on the universal set $X$ are discussed in this portion.

Definition 1 Ashraf et al. (2019) Let $X$ be the non-empty fixed sets. Then, the following set

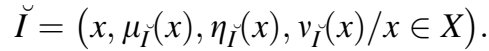

Are said to be Spherical fuzzy set (SFS), where $\mu_{I}(x), \eta_{I}(x), \breve{I_{I}}(x) \in[0,1]$ are called as the grade of membership, positive, neutral, and negative of the elements $x \in$ $X$ to the set $\breve{I}$ respectively, where the following constraint has been fulfilled by $\mu(x), \eta(x), \breve{I}(x)$ for all $x \in X$.

$0 \leq \mu^{2}(x)+\eta^{2}(x)+v^{2}(x) \leq 1$.

Furthermore, $\quad \pi_{I}(x)=\sqrt{1-\left(\mu^{2}(x)+\eta^{2}(x)+v^{2}(x)\right)}$ is referred as the refusal grade of $x \in X$ in $\breve{I}$. For convenience, $\left(\mu_{I}(x), \eta_{I}(x), v_{I}(x)\right)$ is called as an Spherical fuzzy number (SFN). 
Definition 2 Ashraf et al. (2019) Let the three SFNs are $\breve{I}=\left(\mu_{\breve{I}}(x), \eta_{\breve{I}}(x), v_{\breve{I}}(x)\right) \quad \breve{I_{1}}=\left(\mu_{\breve{I_{1}}}(x), \eta_{\breve{I_{1}}}(x), v_{\breve{I_{1}}}(x)\right)$ and $\breve{I_{2}}=\left(\mu_{\breve{I_{2}}}(x), \eta_{\breve{I_{2}}}(x), v_{\breve{I_{2}}}(x)\right)$. And also $\lambda>0$, is any scalar. Then,

(1) $\breve{I^{c}}=\left\{v_{I}(x), \eta_{I}(x), \mu_{I}(x)\right\} ;$

(2) $\breve{I_{1}} \wedge \breve{I_{2}}=\left\{\min \left(\mu_{I_{1}}(x), \mu_{I_{2}}(x)\right)\right.$, $\left.\min \left(\eta_{\breve{I_{1}}}(x), \eta_{\breve{I}_{2}}(x)\right), \max \left(v_{\breve{I_{1}}}(x), v_{\breve{I_{2}}}(x)\right)\right\} ;$

(3) $\breve{I_{1}} \vee \breve{I_{2}}=\left\{\max \left(\mu_{\breve{I_{1}}}(x), \mu_{\breve{I_{2}}}(x)\right)\right.$, $\left.\min \left(\eta_{\breve{I}_{1}}(x), \eta_{\breve{I}_{2}}(x)\right), \min \left(v_{\breve{I}_{1}}(x), v_{\breve{I}_{2}}(x)\right)\right\} ;$

(4) $\breve{I_{1}} \oplus \breve{I_{2}}=\left\{\sqrt{\mu_{\breve{I}_{1}}^{2}(x)+\mu_{\breve{I}_{2}}^{2}(x)-\mu_{\breve{I}_{1}}^{2}(x) \cdot \mu_{\breve{I}_{2}}^{2}}(x), \eta_{\breve{I}_{1}}(x)\right.$. $\left.\eta_{\breve{I_{2}}}(x), v_{\breve{I_{1}}}(x) \cdot v_{\breve{I}_{2}}(x)\right\}$;

(5) $\breve{I_{1}} \otimes \breve{I_{2}}=\left\{\mu_{\breve{I}_{1}}(x) \cdot \mu_{\breve{I_{2}}}(x), \sqrt{\eta_{\breve{I}_{1}}^{2}(x)+\eta_{\breve{I}_{2}}^{2}(x)-\eta_{\breve{I}_{1}}^{2}}\right.$ $\left.(x) \cdot \eta_{\breve{I}_{2}}^{2}(x), \sqrt{v_{I_{1}}^{2}(x)+v_{I_{2}}^{2}(x)-v_{I_{1}}^{2}(x) \cdot v_{I_{2}}^{2}(x)}\right\} ;$

$$
\begin{aligned}
& \lambda \breve{I}=\left\{\sqrt{1-\left(1-\mu_{\breve{I}}^{2}(x)\right)^{\lambda}},\left(\eta_{\breve{I}}(x)\right)^{\lambda},\left(v_{I}(x)\right)^{\lambda}\right\} ; \\
& \left(\breve{I}^{\lambda}=\left\{\left(\mu_{I}(x)\right)^{\lambda}, \sqrt{1-\left(1-\eta_{\breve{I}}^{2}(x)\right)^{\lambda}},\right.\right. \\
& \left.\sqrt{1-\left(1-v_{\breve{I}}^{2}(x)\right)^{\lambda}}\right\} .
\end{aligned}
$$$$
\text { (7) }\left(\breve{I}^{\lambda}=\right.
$$

Definition 3 Garg (2017b) Let $\breve{I}=\left(\mu_{I}(x), \eta_{I}(x), v_{I}(x)\right)$ be the SFN. The score and accuracy function are then described as, follows:

$$
\begin{aligned}
\overline{S c}(\breve{I})= & \mu_{I}(x)-\eta_{I}(x)-v_{I}(x) \\
& \text { where } \overline{S c}(\breve{I}) \in[-1,1], \\
\bar{H} c(\breve{I})= & \mu_{\breve{I}}(x)+\eta_{I}(x)+v_{I}(x) \\
& \text { where } \bar{H} c(\breve{I}) \in[0,1] .
\end{aligned}
$$

Definition 4 Garg (2017b) Let the two SFNs are $\breve{I_{1}}=$ $\left(\mu_{\breve{I}_{1}}(x), \eta_{\breve{I_{1}}}(x), v_{\breve{I}_{1}}(x)\right)$ and $\breve{I_{2}}=\left(\mu_{\breve{I}_{2}}(x), \eta_{\breve{I}_{2}}(x), v_{\breve{I}_{2}}(x)\right)$. Then, the rules for comparison can be defined as if the score function i.e.,

$\therefore \quad \overline{S c}\left(\breve{I_{1}}\right)>\overline{S c}\left(\breve{I_{2}}\right)$, then $\breve{I_{1}}>\breve{I_{2}}$, and if the score function i.e.,

$\therefore \quad \overline{S c}\left(\breve{I_{1}}\right)=\overline{S c}\left(\breve{I_{2}}\right)$, and $\bar{H} c\left(\breve{I_{1}}\right)>\bar{H} c\left(\breve{I_{2}}\right)$, then $\breve{I_{1}}>\breve{I_{2}}$,

$\therefore \quad$ If $\bar{H} c\left(\breve{I_{1}}\right)=\bar{H} c\left(\breve{I_{2}}\right)$, then $\breve{I_{1}}=\breve{I_{2}}$.

\section{New sine trigonometric operational laws (STOLs) for SFSs}

We will define some operational laws for SFNs in this portion.

Definition 5 Let the $S F N$ is $\breve{I}=\left(\mu_{I}(x), \eta_{I}(x), v_{\breve{I}}(x)\right)$. Then, we define a STOLs of a Spherical fuzzy set as;

$$
\begin{aligned}
& \sin \breve{I}=\left\{\sin \left(\frac{\pi}{2}\left(\mu_{I}(x)\right)\right), 2 \sin ^{2}\left(\frac{\pi}{4} \eta_{I}(x)\right),\right. \\
&\left.2 \sin ^{2}\left(\frac{\pi}{4} v_{I}(x)\right)\right\} .
\end{aligned}
$$

From the above definition, it is clear that the $\sin \breve{I}$ is also $S F S$, and also satisfied the following condition of the SFS as, the membership, neutral and nonmembership degrees of $S F S$ are define respectively

$$
\begin{aligned}
\sin \left(\frac{\pi}{2}\left(\mu_{I}(x)\right)\right) & : X \longrightarrow[0,1], \\
\text { such that } 0 & \leq \sin \left(\frac{\pi}{2}\left(\mu_{I}(x)\right)\right) \leq 1, \\
2 \sin ^{2}\left(\frac{\pi}{4} \eta_{I}(x)\right) & : X \longrightarrow[0,1], \\
\text { such that } 0 & \leq 2 \sin ^{2}\left(\frac{\pi}{4} \eta_{I}(x)\right) \leq 1, \\
2 \sin ^{2}\left(\frac{\pi}{4} v_{I}(x)\right) & : X \longrightarrow[0,1], \\
\operatorname{such}(x) & \leq 2 \sin ^{2}\left(\frac{\pi}{4} v_{I}(x)\right) \leq 1,
\end{aligned}
$$

Therefore,

$$
\begin{aligned}
\sin \breve{I}=\{ & \sin \left(\frac{\pi}{2}\left(\mu_{I}(x)\right)\right), 2 \sin ^{2}\left(\frac{\pi}{4} \eta_{I}(x)\right), \\
& \left.2 \sin ^{2}\left(\frac{\pi}{4} v_{I_{I}}(x)\right)\right\}
\end{aligned}
$$

is $S F S$.

Definition 6 Let $\breve{I}=\left(\mu_{I}(x), \eta_{I}(x), v_{I}(x)\right)$ be a $S F N$. Then,

$$
\begin{aligned}
\sin \breve{I}=\{ & \sin \left(\frac{\pi}{2}\left(\mu_{I}(x)\right)\right), 2 \sin ^{2}\left(\frac{\pi}{2} \eta_{I}(x)\right), \\
& \left.2 \sin ^{2}\left(\frac{\pi}{4} v_{I}(x)\right)\right\},
\end{aligned}
$$

is known as sine trigonometric $(S T)$ operator and their value is known as sine trigonometric $S F N$.

Definition 7 Let the collection of SFNs are $\breve{I}=$ $\left(\mu_{I}(x), \eta_{I}(x), v_{\check{I}}(x)\right), \breve{I_{1}}=\left(\mu_{\breve{I_{1}}}(x), \eta_{\breve{I_{1}}}(x), v_{\breve{I_{1}}}(x)\right)$ and $\breve{I_{2}}=$ $\left(\mu_{\breve{I_{2}}}(x), \eta_{\breve{I}_{2}}(x), v_{\breve{I_{2}}}(x)\right)$. Then, we define the following operational laws where $\omega>0$ is any scalar. 
(1)

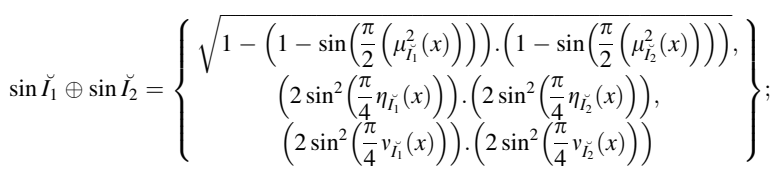

(2)

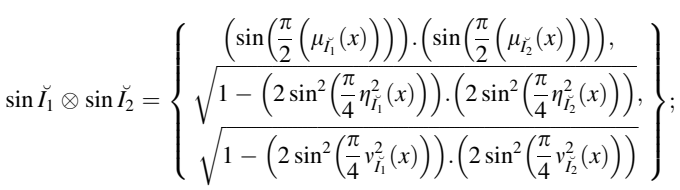

(3)

$$
\begin{aligned}
& \lambda \sin \breve{I}=\left\{\sqrt{1-\left(1-\sin \left(\frac{\pi}{2}\left(\mu_{\breve{I}}^{2}(x)\right)\right)\right)^{\lambda}}\right. \\
& \left.\left(2 \sin ^{2}\left(\frac{\pi}{4} \eta_{\breve{I}}(x)\right)\right)^{\lambda},\left(2 \sin \left(\frac{\pi}{4} v_{\check{I}}(x)\right)\right)^{\lambda}\right\} \\
& (\sin \breve{I})^{\lambda}=\left\{\left(\sin \left(\frac{\pi}{2} \mu_{I}(x)\right)\right)^{\lambda}, \sqrt{1-\left(2 \sin ^{2}\left(\frac{\pi}{4} \eta_{\breve{I}}^{2}(x)\right)\right)^{\lambda}}\right. \\
& \sqrt{\left.1-\left(2 \sin ^{2}\left(\frac{\pi}{4} v_{\breve{I}}^{2}(x)\right)\right)^{\lambda}\right\}}
\end{aligned}
$$$$
\text { (4) }
$$

\subsection{Some basic properties of STOLS of SFNs}

Some fundamental properties of sine trigonometric SFN are discussed in this portion, using the sine trigonometric operational laws (STOLs).

Theorem 1 Let a collection of SFNs are $\breve{I}_{\hat{J}}=$ $\left(\mu_{\breve{I}_{\tilde{J}}}(x), \eta_{\breve{I}_{\tilde{J}}}(x), v_{\breve{I}_{\tilde{J}}}(x)\right)$, where $\hat{J}=1, \ldots, 3$. Then,

(1) $\sin \breve{I_{1}} \oplus \sin \breve{I_{2}}=\sin \breve{I_{2}} \oplus \sin \breve{I_{1}}$

(2) $\sin \breve{I_{1}} \otimes \sin \breve{I_{2}}=\sin \breve{I_{2}} \otimes \sin \breve{I_{1}}$

(3) $\quad\left(\sin \breve{I_{1}} \oplus \sin \breve{I_{2}}\right) \oplus \sin \breve{I_{3}}=\sin \breve{I_{1}} \oplus\left(\sin \breve{I_{2}} \oplus \sin \breve{I_{3}}\right)$

(4) $\left(\sin \breve{I_{1}} \otimes \sin \breve{I_{2}}\right) \otimes \sin \breve{I_{3}}=\sin \breve{I_{1}} \otimes\left(\sin \breve{I_{2}} \otimes \sin \breve{I_{3}}\right)$

Proof Here, we solve the first two parts using the STOLs (sine trigonometric operation laws) define in Definition (7), and the proof of the other two part are similar to the first parts, so we omit here, we get

(1)

$$
\begin{aligned}
& \sin \breve{I_{1}} \oplus \sin \breve{I_{2}}
\end{aligned}
$$

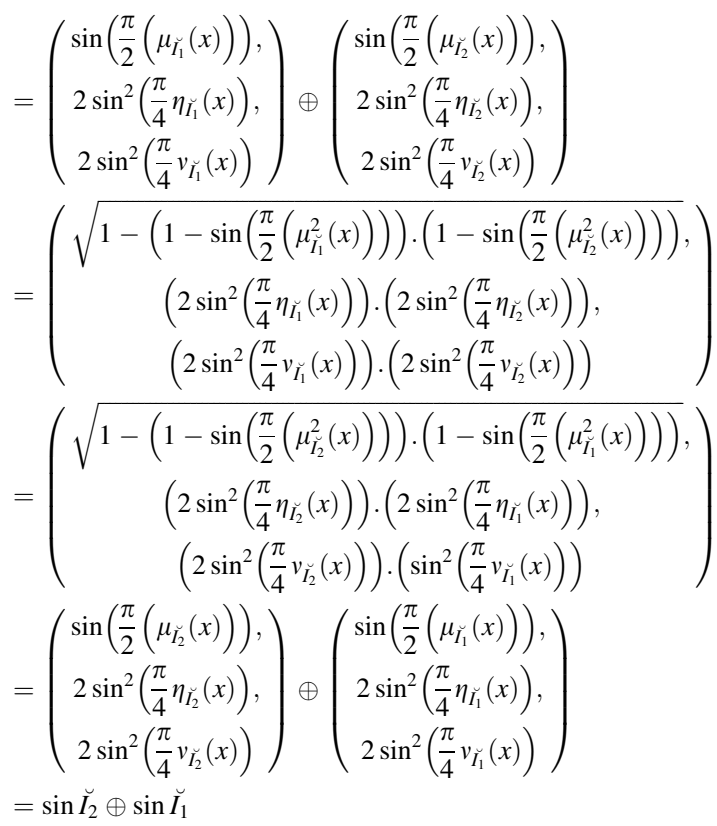

therefore, from the above

$\sin \breve{I_{1}} \oplus \sin \breve{I_{2}}=\sin \breve{I_{2}} \oplus \sin \breve{I_{1}}$

(2)

$$
\begin{aligned}
& \sin \breve{I}_{1} \otimes \sin \breve{I}_{2} \\
& =\left(\begin{array}{c}
\sin \left(\frac{\pi}{2}\left(\mu_{\check{I}_{1}}(x)\right)\right) \\
2 \sin ^{2}\left(\frac{\pi}{4} \eta_{\breve{l}_{1}}(x)\right) \\
\sin ^{2}\left(\frac{\pi}{4} v_{V_{1}}(x)\right)
\end{array}\right)
\end{aligned}
$$

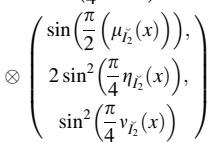

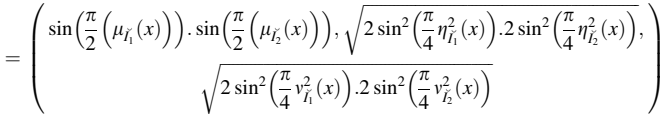

$$
\begin{aligned}
& =\left(\begin{array}{c}
\left.\sin \left(\frac{\pi}{2}\left(\mu_{\xi_{2}}(x)\right)\right) \cdot \sin \left(\frac{\pi}{2}\left(\mu_{\zeta_{1}}(x)\right)\right) \cdot \sqrt{2 \sin ^{2}\left(\frac{\pi}{4} \eta_{h_{i}}^{2}(x)\right) \cdot 2 \sin ^{2}\left(\frac{\pi}{4} \eta_{h_{1}}^{2}(x)\right)}\right) \\
\sqrt{2 \sin ^{2}\left(\frac{\pi}{4} v_{\xi_{2}}^{2}(x)\right) \cdot 2 \sin ^{2}\left(\frac{\pi}{4} v_{h_{1}}^{2}(x)\right)}
\end{array}\right)
\end{aligned}
$$

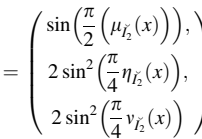

$$
\begin{aligned}
& \otimes\left(\begin{array}{c}
\sin \left(\frac{\pi}{2}\left(\mu_{Y_{1}}(x)\right)\right) \\
2 \sin ^{2}\left(\frac{\pi}{4} \eta_{\eta_{l_{1}}}(x)\right) \\
2 \sin ^{2}\left(\frac{\pi}{4} v_{\zeta_{1}}(x)\right)
\end{array}\right) \\
& =\sin \breve{I}_{1} \otimes \sin \breve{I_{2}}
\end{aligned}
$$

therefore, from the above solution $\sin \breve{I_{1}} \otimes \sin \breve{I_{2}}=\sin \breve{I_{2}} \otimes \sin \breve{I_{1}}$ 
Theorem 2 Let a collection of SFNs are $\breve{I}_{\hat{J}}=$ $\left(\mu_{\breve{I_{\hat{J}}}}(x), \eta_{\breve{I}_{\hat{J}}}(x), v_{\breve{I}_{\hat{J}}}(x)\right)$, where $\hat{J}=1,2$. And also $\lambda, \lambda_{1}, \lambda_{2}>0$ be the real number. Then,

(1) $\lambda\left(\sin \breve{I_{1}} \oplus \sin \breve{I_{2}}\right)=\lambda \sin \breve{I_{1}} \oplus \lambda \sin \breve{I_{2}}$

(2) $\left(\sin \breve{I_{1}} \otimes \sin \breve{I_{2}}\right)^{\lambda}=\left(\sin \breve{I_{1}}\right)^{\lambda} \otimes\left(\sin \breve{I_{2}}\right)^{\lambda}$

(3) $\quad \lambda_{1} \sin \breve{I} \oplus \lambda_{2} \cdot \sin \breve{I}=\left(\lambda_{1} \oplus \lambda_{2}\right) \sin \breve{I}$

(4) $(\sin I)^{\lambda_{1}} \otimes(\sin I)^{\lambda_{2}}=(\sin I)^{\lambda_{1} \oplus \lambda_{2}}$

(5) $\left((\sin I)^{\lambda_{1}}\right)^{\lambda_{2}}=(\sin I)^{\lambda_{1} \cdot \lambda_{2}}$

Proof Here, we will prove the first part of the above theorem only by using the STOLs define in Definition (7), while rest can be proven similarly. As we know,

(1)

$$
\begin{aligned}
\sin \breve{I_{1}}=\{ & \sin \left(\frac{\pi}{2}\left(\mu_{\breve{I_{1}}}(x)\right)\right), 2 \sin ^{2}\left(\frac{\pi}{4} \eta_{\breve{I_{1}}}(x)\right), \\
& \left.2 \sin ^{2}\left(\frac{\pi}{4} v_{\breve{I_{1}}}(x)\right)\right\}
\end{aligned}
$$

and

$$
\begin{aligned}
\sin \breve{I_{2}}=\{ & \sin \left(\frac{\pi}{2}\left(\mu_{\breve{I_{2}}}(x)\right)\right), 2 \sin ^{2}\left(\frac{\pi}{4} \eta_{\breve{I_{2}}}(x)\right), \\
& \left.2 \sin ^{2}\left(\frac{\pi}{4} v_{\breve{I_{2}}}(x)\right)\right\}
\end{aligned}
$$

using the STOLs, we have

$\sin \breve{I_{1}} \oplus \sin \breve{I_{2}}$

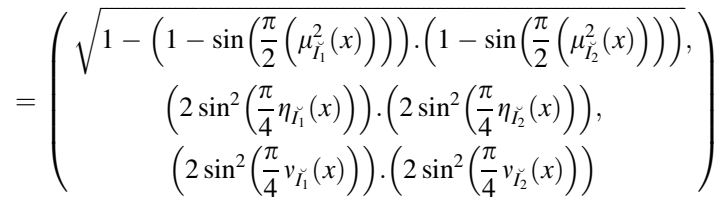

but it is given in statement of the Theorem that $\eta>0$, again we use the Definition (7), we have

$$
\begin{aligned}
& \lambda\left(\sin \breve{I_{1}} \oplus \sin \breve{I_{2}}\right)
\end{aligned}
$$

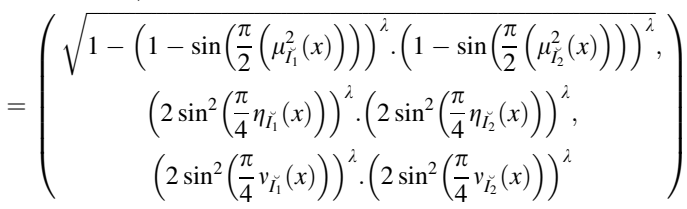

$$
\begin{aligned}
& =\left(\begin{array}{c}
\sqrt{1-\left(1-\sin \left(\frac{\pi}{2}\left(\mu_{I_{1}}^{2}(x)\right)\right)\right)^{\lambda},} \\
\left(2 \sin ^{2}\left(\frac{\pi}{4} \eta_{\breve{I}_{1}}(x)\right)\right)^{\lambda}, \\
\left(2 \sin ^{2}\left(\frac{\pi}{4} v_{\breve{I}_{1}}(x)\right)\right)^{\lambda}
\end{array}\right) \\
& \oplus\left(\begin{array}{c}
\sqrt{1-\left(1-\sin \left(\frac{\pi}{2}\left(\mu_{I_{2}}^{2}(x)\right)\right)\right)^{\lambda},}, \\
\left(2 \sin ^{2}\left(\frac{\pi}{4} \eta_{\breve{I}_{2}}(x)\right)\right)^{\lambda}, \\
2 \sin ^{2}\left(\frac{\pi}{4} v_{\breve{I}_{2}}(x)\right)^{\lambda}
\end{array}\right) \\
& =\lambda \sin \breve{I_{1}} \oplus \lambda \sin \breve{I_{2}}
\end{aligned}
$$

Corollary 1 Let a collection of two SFNs are $\breve{I}_{\hat{J}}=$ $\left(\mu_{\breve{I_{\hat{J}}}}(x), \eta_{\breve{I_{\hat{J}}}}(x), v_{\breve{I_{\hat{J}}}}(x)\right) \quad$ where $\hat{J}=1,2$, such that $\mu_{\breve{I_{1}}}(x) \geq \mu_{\breve{I_{2}}}(x), \eta_{\breve{I_{1}}}(x) \leq \eta_{\breve{I_{2}}}(x) \quad$ and $\quad v_{\breve{I_{1}}}(x) \leq v_{\breve{I_{2}}}(x)$. Then show that $\sin \breve{I_{1}} \geq \sin \breve{I_{2}}$.

Proof Let $\breve{I_{1}}=\left(\mu_{\breve{I_{1}}}(x), \eta_{\breve{I}_{1}}(x), v_{\breve{I_{1}}}(x)\right) \quad$ and $\quad \breve{I_{2}}=$ $\left(\mu_{\breve{I_{2}}}(x), \eta_{\breve{I_{2}}}(x), v_{\breve{I_{2}}}(x)\right)$ are the $\mathrm{SFN}$ with condition $\mu_{1}(x) \geq \mu_{I_{2}}(x)$, since in the closed interval $\left[0, \frac{\pi}{2}\right]$, sin is an increasing function, thus we have $\sin \left(\frac{\pi}{2}\left(\mu_{\breve{I_{1}}}(x)\right)\right) \geq$ $\sin \left(\frac{\pi}{2}\left(\mu_{\breve{I_{2}}}(x)\right)\right)$. But also given that $\eta_{1}(x) \leq \eta_{\breve{I_{2}}}(x)$ which implies that $\left(1-\eta_{\breve{I_{1}}}(x)\right) \geq\left(1-\eta_{\breve{I_{2}}}(x)\right)$, since in closed interval $\left[0, \frac{\pi}{2}\right]$, sine is an increasing function, thus we have $2 \sin ^{2}\left(\frac{\pi}{4} \eta_{\breve{I_{1}}}(x)\right) \geq 2 \sin ^{2}\left(\frac{\pi}{4} \eta_{\breve{I_{2}}}(x)\right)$, which implies that $2 \sin ^{2}\left(\frac{\pi}{4} \eta_{\breve{I_{1}}}(x)\right) \leq 2 \sin ^{2}\left(\frac{\pi}{4} \eta_{\breve{I_{2}}}(x)\right)$, since in closed interval $\left[0, \frac{\pi}{2}\right]$, sin is an increasing function, thus we have $2 \sin ^{2}\left(\frac{\pi}{4} v_{\breve{I_{1}}}(x)\right) \geq 2 \sin ^{2}\left(\frac{\pi}{4} v_{\breve{I_{2}}}(x)\right)$, which implies that $2 \sin ^{2}\left(\frac{\pi}{4} v_{\breve{I_{1}}}(x)\right) \leq 2 \sin ^{2}\left(\frac{\pi}{4} v_{\breve{I_{2}}}(x)\right)$, hence, we get 


$$
\begin{aligned}
& \left(\begin{array}{l}
\sin \left(\frac{\pi}{2}\left(\mu_{\breve{I_{1}}}(x)\right)\right), \\
2 \sin ^{2}\left(\frac{\pi}{4} \eta_{\breve{I_{1}}}(x)\right), \\
2 \sin ^{2}\left(\frac{\pi}{4} v_{\breve{I_{1}}}(x)\right)
\end{array}\right) \\
& \quad\left(\begin{array}{c}
\sin \left(\frac{\pi}{2}\left(\mu_{\breve{I_{2}}}(x)\right)\right), \\
2 \sin ^{2}\left(\frac{\pi}{4} \eta_{\breve{I_{2}}}(x)\right), \\
2 \sin ^{2}\left(\frac{\pi}{4} v_{\breve{I_{2}}}(x)\right)
\end{array}\right)
\end{aligned}
$$

therefore, we get the required result using the Definition (7),

$$
\sin \breve{I_{1}} \geq \sin \breve{I_{2}}
$$

\section{Sine trigonometric aggregation operators}

We have described a number of aggregation operators in this portion of the article on the basis of sine trigonometric operational laws (STOLs).

\subsection{Sine trigonometric averaging aggregation operator}

Definition 8 Let a collection of SFNS are $\breve{I}_{\hat{J}}=$ $\left(\mu_{\breve{I}_{j}}(x), \eta_{\breve{I}_{\hat{J}}}(x), v_{\breve{I}_{j}}(x)\right)$, where $\hat{J}=1, \cdots, n$. Then, the mapping $S T-S F W A: \Psi^{n} \rightarrow \Psi$, is known as the sine trigonometric Spherical fuzzy weighted average $(S T-S F W A)$ operator, if

$$
\begin{aligned}
S T & -S F W A\left(\breve{I_{1}}, \cdots, \breve{I_{n}}\right) \\
& =\omega_{1} \cdot \sin \breve{\breve{I}_{1}} \oplus \cdots \oplus \omega_{n} \cdot \sin \breve{I_{n}} .
\end{aligned}
$$

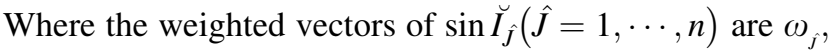
which fulfilled the criteria of $\omega_{\hat{J}}>0$, and $\sum_{\hat{J}=1}^{n} \omega_{\hat{J}}=1$.

Theorem 3 Let a collection of SFNs are $\breve{I_{\hat{J}}}=$ $\left(\mu_{\breve{I}_{j}}(x), \eta_{\breve{I}_{\hat{J}}}(x), v_{\breve{I}_{j}}(x)\right)$, where $\hat{J}=1, \cdots, n$. Then, the aggregated value is also SFN by utilizing the ST - SFWA operator, and is given by

$$
\begin{aligned}
S T & -S F W A\left(\breve{I_{1}}, \cdots, \breve{I_{n}}\right) \\
= & \left(\begin{array}{c}
\sqrt{1-\prod_{\hat{J}=1}^{n}\left(1-\sin \left(\frac{\pi}{2}\left(\mu_{\hat{J}}^{2}(x)\right)\right)\right)^{\omega_{\hat{j}}}}, \\
\prod_{\hat{J}=1}^{n}\left(2 \sin ^{2}\left(\frac{\pi}{4} \eta_{\hat{J}}(x)\right)\right)^{\omega_{\hat{J}}}, \\
\prod_{\hat{J}=1}^{n}\left(2 \sin ^{2}\left(\frac{\pi}{4} v_{\hat{J}}(x)\right)\right)^{\omega_{\hat{J}}}
\end{array}\right)
\end{aligned}
$$

Proof Using the process of mathematical induction, we prove the said Theorem. Because, $\breve{I_{\hat{J}}}=$ $\left(\mu_{\breve{I}_{f}}(x), \eta_{\breve{I}_{\tilde{f}}}(x), v_{\breve{I}_{f}}(x)\right)$ is $S F N$ for each $\hat{J}$, which implies that $\quad\left(\mu_{\breve{I}_{j}}(x), \eta_{\breve{I}_{j}}(x), v_{\breve{I}_{j}}(x)\right) \in[0,1] \quad$ and $\quad$ also $\left(\mu_{I_{j}}^{2}(x)+\eta_{\check{I}_{j}}^{2}(x)+v_{I_{j}}^{2}(x)\right) \leq 1$. The following mathematical induction steps were then performed.

Step 1. Now for $n=2$, we get $S T-S F W A\left(\breve{I_{1}}, \breve{I_{2}}\right)=\omega_{1} \cdot \sin \breve{I_{1}} \oplus \omega_{2} \cdot \sin \breve{I_{2}}$

where

$$
=\left(\begin{array}{c}
\omega_{1} \sin \breve{I_{1}} \\
\left(2 \sin ^{2}\left(\frac{\pi}{2} v_{\breve{I}_{1}}(x)\right)\right)^{\omega_{1}}
\end{array}\right)
$$

and

$\omega_{2} \sin \breve{I_{2}}$

$$
=\left(\begin{array}{c}
\sqrt{1-\left(1-\sin \left(\frac{\pi}{2}\left(\mu_{I_{2}}^{2}(x)\right)\right)\right)^{\omega_{2}}},\left(2 \sin ^{2}\left(\frac{\pi}{2} \eta_{\check{I}_{2}}(x)\right)\right)^{\omega_{2}}, \\
\left(2 \sin ^{2}\left(\frac{\pi}{2} v_{\check{I}_{2}}(x)\right)\right)^{\omega_{2}}
\end{array}\right)
$$

and hence, using the Definition in (7), we get $\omega_{1} \sin \breve{I_{1}} \oplus \omega_{2} \sin \breve{I_{2}}$

$$
=\left(\begin{array}{c}
\sqrt{1-\prod_{\hat{J}=1}^{2}\left(1-\sin \left(\frac{\pi}{2}\left(\mu_{\hat{J}}^{2}(x)\right)\right)\right)^{\omega_{\hat{J}}}}, \\
\prod_{\hat{J}=1}^{2}\left(2 \sin ^{2}\left(\frac{\pi}{4} \eta_{\hat{J}}(x)\right)\right)^{\omega_{\hat{J}}}, \\
\prod_{\hat{J}=1}^{2}\left(2 \sin ^{2}\left(\frac{\pi}{4} v_{\hat{J}}(x)\right)\right)^{\omega_{\hat{J}}}
\end{array}\right)
$$

Step 2. Now, say it is true for $n=k$. 


$$
\begin{aligned}
S T & -S F W A\left(\breve{I_{1}}, \breve{I_{2}}\right) \\
& =\left(\begin{array}{c}
\sqrt{1-\prod_{\hat{J}=1}^{k}\left(1-\sin \left(\frac{\pi}{2}\left(\mu_{\hat{J}}^{2}(x)\right)\right)\right)^{\omega_{j}}}, \\
\prod_{\hat{J}=1}^{k}\left(2 \sin ^{2}\left(\frac{\pi}{4} \eta_{\hat{J}}(x)\right)\right)^{\omega_{\hat{J}}}, \\
\prod_{\hat{J}=1}^{k}\left(2 \sin ^{2}\left(\frac{\pi}{4} v_{\hat{J}}(x)\right)\right)^{\omega_{\hat{J}}}
\end{array}\right)
\end{aligned}
$$

Step 3. Now, we prove that this is true for $n=k+1$

$$
\begin{aligned}
S T & -S F W A\left(\breve{I_{1}}, \cdots, \breve{I_{k+1}}\right) \\
= & \omega_{1} \sin \breve{I_{1}} \oplus \cdots \oplus \omega_{n} \sin \breve{I_{n}} \oplus \omega_{k+1} \sin \breve{I_{k+1}} \\
& =\left(\begin{array}{c}
\sqrt{1-\prod_{\hat{J}=1}^{k}\left(1-\sin \left(\frac{\pi}{2}\left(\mu_{\hat{J}}^{2}(x)\right)\right)\right)^{\omega_{\hat{J}}}}, \\
\prod_{\hat{J}=1}^{k}\left(2 \sin \left(\frac{\pi}{4} \eta_{\hat{J}}(x)\right)\right)^{\omega_{j}}, \\
\prod_{\hat{J}=1}^{k}\left(2 \sin ^{2}\left(\frac{\pi}{4} v_{\hat{J}}(x)\right)\right)^{\omega_{\hat{J}}}
\end{array}\right) \\
& \left(\begin{array}{c}
\sqrt{1-\left(1-\sin \left(\frac{\pi}{2}\left(\mu_{k+1}^{2}(x)\right)\right)\right)^{\omega_{k+1}}}, \\
\left(2 \sin ^{2}\left(\frac{\pi}{4} \eta_{k+1}(x)\right)\right)^{\omega_{k+1}}, \\
\left(2 \sin ^{2}\left(\frac{\pi}{4} v_{k+1}(x)\right)\right)^{\omega_{k+1}}
\end{array}\right)
\end{aligned}
$$

again, using the Definition (7), we obtained

$$
\begin{aligned}
S T & -S F W A\left(\breve{I_{1}}, \cdots, \breve{I_{k+1}}\right) \\
= & \left(\begin{array}{c}
\sqrt{1-\prod_{\hat{J}=1}^{k+1}\left(1-\sin \left(\frac{\pi}{2}\left(\mu_{\hat{J}}^{2}(x)\right)\right)\right)^{\omega_{\hat{j}}}}, \\
\prod_{\hat{J}=1}^{k+1}\left(2 \sin ^{2}\left(\frac{\pi}{4} \eta_{\hat{J}}(x)\right)\right)^{\omega_{\hat{J}}}, \\
\prod_{\hat{J}=1}^{k+1}\left(2 \sin ^{2}\left(\frac{\pi}{4} v_{\hat{J}}(x)\right)\right)^{\omega_{\hat{J}}}
\end{array}\right)
\end{aligned}
$$

Hence, for the $n=k+1$ holds. Then, the statement is valid for all $n$ through the principal of mathematical induction.

The $S T-S F W A$ operators possess the following properties.

Property 1 If all collection of SFNS $\breve{I_{\hat{J}}}=\breve{I}$, where $\breve{I}$ is another $\operatorname{SFN}(\hat{J}=1, \cdots, n)$, then

$S T-S F W A\left(\breve{I_{1}}, \cdots, \breve{I_{n}}\right)=\sin \breve{I}$

Proof Let $\breve{I}=\left(\mu_{I}(x), \eta_{I}(x), v_{I}(x)\right)$ is $S F N$, such that $\breve{I_{\hat{J}}}=\breve{I}$. Then, we get using Theorem (3),

$$
\begin{aligned}
& S T-S F W A\left(\breve{I_{1}}, \cdots, \breve{I_{n}}\right) \\
& =\left(\begin{array}{c}
\sqrt{1-\prod_{\hat{J}=1}^{n}\left(1-\sin \left(\frac{\pi}{2}\left(\mu_{\hat{J}}^{2}(x)\right)\right)\right)^{\omega_{\hat{J}}}}, \\
\prod_{\hat{J}=1}^{n}\left(2 \sin ^{2}\left(\frac{\pi}{4} \eta_{\hat{J}}(x)\right)\right)^{\omega_{\hat{f}}}, \\
\prod_{\hat{J}=1}^{n}\left(2 \sin ^{2}\left(\frac{\pi}{4} v_{\hat{J}}(x)\right)\right)^{\omega_{\hat{J}}}
\end{array}\right) \\
& =\left(\begin{array}{c}
\sqrt{1-\prod_{\hat{J}=1}^{n}\left(1-\sin \left(\frac{\pi}{2}\left(\mu^{2}(x)\right)\right)\right)^{\omega_{\hat{J}}}}, \\
\prod_{\hat{J}=1}^{n}\left(2 \sin \left(\frac{\pi}{4} \eta(x)\right)\right)^{\omega_{\hat{J}}}, \\
\prod_{\hat{J}=1}^{n}\left(2 \sin ^{2}\left(\frac{\pi}{4} v(x)\right)\right)^{\omega_{\hat{J}}}
\end{array}\right) \\
& =\left(\begin{array}{c}
\sqrt{1-\left(1-\sin \left(\frac{\pi}{2}\left(\mu^{2}(x)\right)\right)\right)^{\sum_{j=1}^{n} \omega_{j}}}, \\
\left(2 \sin ^{2}\left(\frac{\pi}{4} \eta(x)\right)\right)^{\sum_{j=1}^{n} \omega_{f}}, \\
\left(2 \sin \left(\frac{\pi}{4} v(x)\right)\right)^{\sum_{j=1}^{n} \omega_{f}}
\end{array}\right) \\
& =\left(\begin{array}{c}
\sin \left(\frac{\pi}{2}(\mu(x))\right),\left(2 \sin \left(\frac{\pi}{4} \eta(x)\right)\right) \\
\left(2 \sin ^{2}\left(\frac{\pi}{4} v(x)\right)\right)
\end{array}\right)
\end{aligned}
$$$$
=\sin \breve{I}
$$

Property 2 If $\quad \breve{I_{\hat{J}}}=\left(\mu_{\breve{I}_{\hat{J}}}(x), \eta_{\breve{I}_{\hat{J}}}(x), v_{\breve{I_{j}}}(x)\right), \breve{I_{\hat{J}}^{-}}=$ $\left(\min _{\hat{J}}\left\{\mu_{\hat{J}}(x)\right\}, \max _{\hat{J}}\left\{\eta_{\hat{J}}(x)\right\}, \max _{\hat{J}}\left\{v_{\hat{J}}(x)\right\}\right)$ and

$$
\breve{I_{\hat{J}}^{+}}=\left(\max _{\hat{J}}\left\{\mu_{\hat{J}}(x)\right\}, \min _{\hat{J}}\left\{\eta_{\hat{J}}(x)\right\}, \min _{\hat{J}}\left\{v_{\hat{J}}(x)\right\}\right),
$$

where $\hat{J}=1, \cdots, n$, be SFNs, then

$\sin \breve{I^{-}} \leq S T-S F W A\left(\breve{I_{1}}, \cdots, \breve{I_{n}}\right) \leq \sin \breve{I}^{+}$.

Proof Since for any $\hat{J}$, $\min _{\hat{J}}\left\{\mu_{\hat{J}}(x)\right\} \leq \mu_{\hat{J}}(x) \leq \max _{\hat{J}}\left\{\mu_{\hat{J}}(x)\right\}$,

$\min _{\hat{J}}\left\{\eta_{\hat{J}}(x)\right\} \leq \eta_{\hat{J}}(x) \leq \max _{\hat{J}}\left\{\eta_{\hat{J}}(x)\right\} \quad$ and $\min _{\hat{J}}\left\{v_{\hat{J}}(x)\right\} \leq v_{\hat{J}}(x) \leq \max _{\hat{J}}\left\{v_{\hat{J}}(x)\right\}$. This implies that $\breve{I^{-}} \leq \breve{I_{\hat{J}}} \leq \breve{I^{+}}$. Assume that,

$$
S T-S F W A\left(\breve{I_{1}}, \cdots, \breve{I_{n}}\right)=\sin \breve{I}=\left(\mu_{I}(x), \eta_{I}(x), v_{I}(x)\right),
$$$$
\sin \breve{I^{+}}=\left(\mu_{I^{+}}(x), \eta_{I^{-}}(x), v_{\check{I}^{-}}(x)\right) \quad \text { and } \quad \sin \breve{I^{-}}=
$$ 
$\left(\mu_{I^{-}}(x), \eta_{I^{+}}(x), v_{I^{+}}(x)\right)$. Then, by the monotonicity of the sine trigonometric function, we have

$$
\begin{aligned}
& \mu_{I}(x)=\sqrt{1-\prod_{\hat{J}=1}^{n}\left(1-\sin \left(\frac{\pi}{2}\left(\mu_{\hat{J}}^{2}(x)\right)\right)\right)^{\omega_{\hat{f}}}} \\
& \geq \sqrt{1-\prod_{\hat{J}=1}^{n}\left(1-\sin \left(\frac{\pi}{2} \min _{\hat{J}}\left\{\mu_{\hat{J}}^{2}(x)\right\}\right)\right)^{\omega_{\hat{J}}}} \\
& =\sqrt{1-\left(1-\sin \left(\frac{\pi}{2} \min \left\{\mu_{\hat{J}}^{2}(x)\right\}\right)\right)^{\sum_{j=1}^{n} \omega_{f}}} \\
& =\sin \left(\frac{\pi}{2} \min \left\{\mu_{J}(x)\right\}\right) \\
& =\mu_{I^{-}}(x) \\
& \eta_{I_{I}}(x)=\prod_{\hat{J}=1}^{n}\left(2 \sin \left(\frac{\pi}{4} \eta_{\hat{J}}(x)\right)\right)^{\omega_{\hat{J}}} \\
& \geq \prod_{\hat{J}=1}^{n}\left(2 \sin ^{2}\left(\frac{\pi}{4} \min _{\hat{J}}\left\{\eta_{\hat{J}}(x)\right\}\right)\right)^{\omega_{\hat{J}}} \\
& =\left(2 \sin ^{2}\left(\frac{\pi}{4} \min \left\{\eta_{\hat{J}}(x)\right\}\right)\right)^{\sum_{j=1}^{n} \omega_{\hat{J}}} \\
& =\left(2 \sin ^{2}\left(\frac{\pi}{4} \min _{\hat{J}}\left\{\eta_{\hat{J}}(x)\right\}\right)\right) \\
& =\eta_{I^{-}}(x) \\
& v_{I}(x)=\prod_{\hat{J}=1}^{n}\left(2 \sin ^{2}\left(\frac{\pi}{4} v_{\hat{J}}(x)\right)\right)^{\omega_{j}} \\
& \geq \prod_{\hat{J}=1}^{n}\left(2 \sin ^{2}\left(\frac{\pi}{4} \min _{\hat{J}}\left\{v_{\hat{J}}(x)\right\}\right)\right)^{\omega_{\hat{J}}} \\
& =\left(2 \sin ^{2}\left(\frac{\pi}{4} \min \left\{v_{\hat{J}}(x)\right\}\right)\right)^{\sum_{\hat{j}=1}^{n} \omega_{\hat{J}}} \\
& =\left(2 \sin ^{2}\left(\frac{\pi}{4} \min _{\hat{J}}\left\{v_{\hat{J}}(x)\right\}\right)\right) \\
& =v_{\check{I}^{-}}(x)
\end{aligned}
$$

and also

$$
\begin{aligned}
& \mu_{I}(x)=\sqrt{1-\prod_{\hat{J}=1}^{n}\left(1-\sin \left(\frac{\pi}{2}\left(\mu_{\hat{J}}^{2}(x)\right)\right)\right)^{\omega_{f}}} \\
& \leq \sqrt{1-\prod_{\hat{J}=1}^{n}\left(1-\sin \left(\frac{\pi}{2} \max _{\hat{J}}\left\{\mu_{\hat{J}}^{2}(x)\right\}\right)\right)^{\omega_{\hat{J}}}} \\
& =\sqrt{1-\left(1-\sin \left(\frac{\pi}{2} \max \left\{\mu_{\hat{J}}^{2}(x)\right\}\right)\right)^{\sum_{j=1}^{n} \omega_{j}}} \\
& =\sin \left(\frac{\pi}{2} \max _{\hat{J}}\left\{\mu_{\hat{J}}(x)\right\}\right) \\
& =\mu_{I^{+}}(x) \\
& \eta_{I}(x)=\prod_{\hat{J}=1}^{n}\left(2 \sin ^{2}\left(\frac{\pi}{4} \eta_{\hat{J}}(x)\right)\right)^{\omega_{\hat{J}}} \\
& \leq \prod_{\hat{J}=1}^{n}\left(2 \sin ^{2}\left(\frac{\pi}{4} \max _{\hat{J}}\left\{\eta_{\hat{J}}(x)\right\}\right)\right)^{\omega_{\hat{J}}} \\
& =\left(2 \sin ^{2}\left(\frac{\pi}{4} \max \left\{\eta_{\hat{J}}(x)\right\}\right)\right)^{\sum_{\hat{J}=1}^{n} \omega_{\hat{J}}} \\
& =\left(2 \sin ^{2}\left(\frac{\pi}{4} \max _{\hat{J}}\left\{\eta_{\hat{J}}(x)\right\}\right)\right) \\
& =\eta_{I^{+}}(x) \\
& v_{I}(x)=\prod_{\hat{J}=1}^{n}\left(2 \sin ^{2}\left(\frac{\pi}{4} v_{\hat{J}}(x)\right)\right)^{\omega_{\hat{J}}} \\
& \geq \prod_{\hat{J}=1}^{n}\left(2 \sin ^{2}\left(\frac{\pi}{4} \max _{\hat{J}}\left\{v_{\hat{J}}(x)\right\}\right)\right)^{\omega_{\hat{J}}} \\
& =\left(2 \sin ^{2}\left(\frac{\pi}{4} \max \left\{v_{\hat{J}}(x)\right\}\right)\right)^{\sum_{\hat{f}=1}^{n} \omega_{\hat{J}}} \\
& =\left(2 \sin ^{2}\left(\frac{\pi}{4} \max _{\hat{J}}\left\{v_{\hat{J}}(x)\right\}\right)\right) \\
& =v_{I^{+}}(x)
\end{aligned}
$$

Based on score function Definition (3), we get

$$
\begin{aligned}
& S c(\sin \breve{I})=\mu_{I}(x)-\eta_{I}(x)-v_{\check{I}}(x)
\end{aligned}
$$

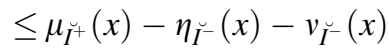

$$
\begin{aligned}
& =S c\left(\sin \breve{I}^{+}\right) \\
& S c(\sin \breve{I})=\mu_{I}(x)-\eta_{I}(x)-v_{I}(x) \\
& \geq \mu_{I^{-}}(x)-\eta_{I^{+}}(x)-v_{I^{+}}(x) \\
& =S c\left(\sin \breve{I^{-}}\right)
\end{aligned}
$$

Hence, $S c\left(\sin \breve{I^{-}}\right) \leq S c(\sin \breve{I}) \leq S c\left(\sin \breve{I}^{+}\right)$.

Now, we have explain three cases:

Case 1 If $S c\left(\sin \breve{I^{-}}\right) \leq S c(\sin \breve{I}) \leq S c\left(\sin \breve{I^{+}}\right)$, then result holds. 
Case 2 If $S c\left(\sin \breve{I}^{+}\right)=S c(\sin \breve{I})$ then

$$
\mu_{I}(x)-\eta_{I^{\prime}}(x)-v_{I^{\prime}}(x)=\mu_{I^{+}}(x)-\eta_{I^{+}}(x)-v_{I^{+}}(x),
$$

which implies that $\mu_{I}(x)=\mu_{I^{+}}(x), \eta_{I^{\prime}}(x)=\eta_{I^{+}}(x)$, and $v_{I}(x)=v_{I^{+}}(x)$ and $H\left(\sin \breve{I^{+}}\right)=H(\sin \breve{I})$.

Case 3 If $S c\left(\sin \breve{I}^{-}\right)=S c(\sin \breve{I})$, then

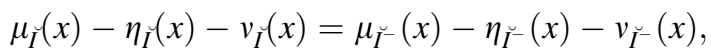

which implies that $\mu_{I}(x)=\mu_{\breve{I^{-}}}(x), \eta_{I^{\prime}}(x)=\eta_{I^{-}}(x)$, and $v_{I^{\prime}}(x)=v_{I^{-}}(x)$ and $H\left(\sin \breve{I}^{-}\right)=H(\sin \breve{I})$, therefore, by combining all these cases, we get

$\sin \breve{I}^{-} \leq S T-S F W A\left(\breve{I_{1}}, \cdots, \breve{I_{n}}\right) \leq \sin \breve{I}^{+}$

Property 3 Let the collection of SFNs are $\breve{I}_{\hat{J}}=$ $\left(\mu_{\breve{J}_{\hat{J}}}(x), \eta_{\breve{I}_{\hat{J}}}(x), v_{\breve{I}_{\hat{J}}}(x)\right)$ and

$$
{\breve{I_{\hat{J}}}}_{\hat{J}}^{*}=\left(\mu_{I_{\hat{J}}}^{*}(x), \eta_{I_{\hat{J}}}^{*}(x), v_{I_{\hat{J}}}^{*}(x)\right),
$$

where $\hat{J}=1, \cdots, n$. If

$$
\mu_{\breve{I_{\hat{J}}}}(x) \leq \mu_{\breve{I}_{\hat{J}}}^{*}(x), \eta_{\check{I}_{\hat{J}}}(x) \geq \eta_{\breve{I}_{\hat{J}}}^{*}(x)
$$

and $v_{I_{\hat{J}}}(x) \geq v_{\breve{I}_{\hat{J}}}^{*}(x)$, then

$S T-S F W A\left(\breve{I_{1}}, \cdots, \breve{I_{n}}\right) \leq S T-S F W A\left(\breve{I}_{1}^{*}, \cdots, \breve{I}_{n}^{*}\right)$.

Proof Follow from the above, so we omit here.

Definition 9 A sine trigonometric $S F$ ordered weighted average operator $(S T-S F O W A)$ is a mapping $S T-$ SFOWA $: \Psi^{n} \rightarrow \Psi$ such that weighted vector $\omega=$ $\left(\omega_{1}, \cdots, \omega_{n}\right)^{T}$, which fulfilled the criteria of $\omega_{\hat{J}}>0$ and $\sum_{\hat{J}=1}^{n} \omega_{\hat{J}}=1$.

$S T-S F O W A=\omega_{1} \sin \breve{I}_{\sigma(1)} \oplus \cdots \oplus \omega_{n} \sin \breve{I}_{\sigma(n)}$.

Where $(1, \cdots, n)$ is the permutation $\sigma$, such that $\breve{I}_{\sigma(\hat{J}-1)} \geq \breve{I}_{\sigma(\hat{J})}$ for any $\hat{J}$.

Theorem 4 Let a collection of SFNs are $\breve{I}_{\hat{J}}=$ $\left(\mu_{\breve{I}_{\hat{J}}}(x), \eta_{\breve{I}_{\hat{j}}}(x), v_{\breve{I}_{\hat{J}}}(x)\right)$, where $\hat{J}=1, \cdots, n$. Then, by utilizing the operator i.e., ST - SFOWA the aggregated value is also SFN and is given by,

$$
\begin{gathered}
S T-\operatorname{SFOWA}\left(\breve{I_{1}}, \cdots, \breve{I_{n}}\right) \\
=\left(\begin{array}{c}
\sqrt{1-\prod_{\hat{J}=1}^{n}\left(1-\sin \left(\frac{\pi}{2}\left(\mu_{\sigma(\hat{J})}^{2}(x)\right)\right)\right)^{\omega_{\hat{J}}}}, \\
\prod_{\hat{J}=1}^{n}\left(2 \sin ^{2}\left(\frac{\pi}{4} \eta_{\sigma(\hat{J})}(x)\right)\right)^{\omega_{\hat{J}}}, \\
\prod_{\hat{J}=1}^{n}\left(2 \sin ^{2}\left(\frac{\pi}{4} v_{\sigma(\hat{J})}(x)\right)\right)^{\omega_{\hat{J}}}
\end{array}\right) .
\end{gathered}
$$

Proof Proof is same to Theorem (3), so proof is ignore here.

Definition 10 A sine trigonometric $S F$ hybrid average operator $(S T-S F H A)$ is a mapping $S T-S F H A: \Psi^{n} \rightarrow$ $\Psi$ such that the associate vectors $\xi=\left(\xi_{1}, \xi_{2}, \cdots, \xi_{n}\right)^{T}$, which fulfilled the criteria of $\xi_{\hat{J}}>0$ and $\sum_{\hat{J}=1}^{n} \xi_{\hat{J}}=1$.

$S T-S F H A=\xi_{1} \sin \ddot{I}_{\sigma(1)} \oplus \cdots \oplus \xi_{n} \sin \ddot{I}_{\sigma(n)}$.

Where $(1, \cdots, n)$ is the permutation of $\sigma$, as $\breve{I}_{\sigma(\hat{J}-1)} \geq \breve{I}_{\sigma(\hat{J})}$ for any $\hat{J}$ and $\ddot{I}_{\hat{J}}=n \omega_{\hat{J}} \breve{I}_{\hat{J}}$

Theorem 5 Let a collection of SFNs are $\breve{I}_{\hat{J}}=$ $\left(\mu_{\breve{I_{\hat{J}}}}(x), \eta_{\breve{I_{\hat{J}}}}(x), v_{\breve{I_{\hat{J}}}}(x)\right)$, where $\hat{J}=1, \cdots, n$. Then, the aggregated value is also SFN by utilized the operator STSFHA and is given by,

$$
\begin{aligned}
& S T-\operatorname{SFHA}\left(\breve{I_{1}}, \cdots, \breve{I_{n}}\right) \\
& =\left(\begin{array}{c}
\sqrt{1-\prod_{\hat{J}=1}^{n}\left(1-\sin \left(\frac{\pi}{2}\left(\tilde{\mu}_{\sigma(\hat{J})}^{2}(x)\right)\right)\right)^{\omega_{\hat{J}}}}, \\
\prod_{\hat{J}=1}^{n}\left(2 \sin ^{2}\left(\frac{\pi}{4} \widetilde{\eta}_{\sigma(\hat{J})}(x)\right)\right)^{\omega_{\hat{J}}}, \\
\prod_{\hat{J}=1}^{n}\left(2 \sin ^{2}\left(\frac{\pi}{4} \widetilde{v}_{\sigma(\hat{J})}(x)\right)\right)^{\omega_{\hat{J}}}
\end{array}\right) .
\end{aligned}
$$

Proof Proof is same to Theorem (3), so proof is ignore here.

\subsection{Sine trigonometric geometric aggregation operator}

Definition 11 Let a collection of SFNS are $\breve{I}_{\hat{J}}=$ $\left(\mu_{\breve{I_{\hat{J}}}}(x), \eta_{\breve{I_{\hat{J}}}}(x), v_{\breve{I_{\hat{J}}}}(x)\right)$, where $\hat{J}=1, \cdots, n$. Then, the mapping $S T-S F W G: \Psi^{n} \rightarrow \Psi$, is known as the sine trigonometric Spherical fuzzy weighted geometric $(S T-S F W G)$ operator, if 


$$
\begin{aligned}
S T & -S F W G\left(\breve{I_{1}}, \cdots, \breve{I_{n}}\right) \\
& =\left(\sin \breve{I_{1}}\right)^{\omega_{1}} \otimes \ldots \otimes\left(\sin \breve{I_{n}}\right)^{\omega_{1}} .
\end{aligned}
$$

Where the weight vectors are $\omega=\left(\omega_{1}, \cdots, \omega_{n}\right)^{T}$ of $\sin \breve{I_{\hat{J}}}(\hat{J}=1, \cdots, n)$, which fulfilled the criteria of $\omega_{\hat{J}}>0$, and $\sum_{\hat{J}=1}^{n} \omega_{\hat{J}}=1$.

Theorem 6 Let a collection of SFNs are $\breve{I_{\hat{J}}}=$ $\left(\mu_{\breve{I}_{j}}(x), \eta_{\breve{I}_{j}}(x), v_{\breve{I}_{j}}(x)\right)$, where $\hat{J}=1, \cdots, n$. Then, the aggregated value is also SFN by using the $S T-S F W G$ operator, and is given by,

$$
\begin{aligned}
S T & -S F W G\left(\breve{I_{1}}, \cdots, \breve{I_{n}}\right) \\
& =\left(\begin{array}{c}
\prod_{\hat{J}=1}^{n}\left(\sin \left(\frac{\pi}{2}\left(\mu_{\hat{J}}(x)\right)\right)\right)^{\omega_{\hat{J}}}, \\
\sqrt{1-\prod_{\hat{J}=1}^{n}\left(2 \sin ^{2}\left(\frac{\pi}{4} \eta_{\hat{J}}^{2}(x)\right)\right)^{\omega_{\hat{J}}}}, \\
\sqrt{1-\prod_{\hat{J}=1}^{n}\left(2 \sin ^{2}\left(\frac{\pi}{4} v_{\hat{J}}^{2}(x)\right)\right)^{\omega_{\hat{J}}}}
\end{array}\right) .
\end{aligned}
$$

Proof Proof is similar to Theorem (3), so procedure is ignore here.

The $S T-S F W G$ operators possess the following properties.

Property 1 If all collection of SFNs $\breve{I_{\hat{J}}}=\breve{I}$, where $\breve{I}$ is another $\operatorname{SFN}(\hat{J}=1, \cdots, n)$, then

$S T-S F W G\left(\breve{I_{1}}, \cdots, \breve{I_{n}}\right)=\sin \breve{I}$

Property 2 If $\breve{I_{\hat{J}}}=\left(\mu_{\breve{I}_{\hat{J}}}(x), \eta_{\check{I}_{\hat{J}}}(x), v_{\breve{I}_{\hat{J}}}(x)\right)$, where $\hat{J}=$ $1, \cdots, n$,

$$
\breve{I_{\hat{J}}^{-}}=\left(\min _{\hat{J}}\left\{\mu_{\hat{J}}(x)\right\}, \max _{\hat{J}}\left\{\eta_{\hat{J}}(x)\right\}, \max _{\hat{J}}\left\{v_{\hat{J}}(x)\right\}\right)
$$

and

$$
\breve{I_{\hat{J}}^{+}}=\left(\max _{\hat{J}}\left\{\mu_{\hat{J}}(x)\right\}, \min _{\hat{J}}\left\{\eta_{\hat{J}}(x)\right\}, \min _{\hat{J}}\left\{v_{\hat{J}}(x)\right\}\right)
$$

be SFNs, then

$\sin \breve{I^{-}} \leq S T-S F W G\left(\breve{I_{1}}, \cdots, \breve{I_{n}}\right) \leq \sin \breve{I}^{+}$.

Property 3 Let the collection of SFNS are $\breve{I}_{\hat{J}}=$ $\left(\mu_{\breve{I}_{j}}(x), \eta_{\breve{I}_{j}}(x), v_{\breve{I}_{j}}(x)\right)$ and

$$
\breve{I_{J}^{*}}=\left(\mu_{I_{f}}^{*}(x), \eta_{I_{\tilde{J}}}^{*}(x), v_{I_{f}}^{*}(x)\right),
$$

where $\hat{J}=1, \cdots, n$. If

$$
\mu_{\breve{I}_{j}}(x) \leq \mu_{I_{j}}^{*}(x), \eta_{\breve{I}_{j}}(x) \geq \eta_{I_{j}}^{*}(x),
$$

and $v_{\breve{I}_{\tilde{J}}}(x) \geq v_{\breve{I}_{\breve{f}}}^{*}(x)$. Then,

$S T-S F W G\left(\breve{I_{1}}, \cdots, \breve{I_{n}}\right) \leq S T-S F W A\left(\breve{I}_{1}^{*}, \cdots, \breve{I_{n}^{*}}\right)$.

Definition 12 A $S T-S F O W G$ is a mapping from $\Psi^{n}$ to $\Psi$ such that the weighted vector $\omega=\left(\omega_{1}, \cdots, \omega_{n}\right)^{T}$, which fulfilled the criteria of $\omega_{\hat{J}}>0$ and $\sum_{\hat{J}=1}^{n} \omega_{\hat{J}}=1$.

$$
S T-S F O W G=\left(\operatorname { s i n } { \breve { I _ { \sigma ( 1 ) } } } ^ { \omega _ { 1 } } \oplus \cdots \oplus \left(\sin {\breve{I_{\sigma(n)}}}^{\omega_{n}} .\right.\right.
$$

Where $\sigma$ is the permutation of $(1, \cdots, n)$ as $\breve{I}_{\sigma(\hat{J}-1)} \geq \breve{I}_{\sigma(\hat{J})}$ for any $\hat{J}$.

Theorem 7 Let a family of SFNs are ${\breve{I_{\hat{J}}}}=$ $\left(\mu_{\breve{I}_{j}}(x), \eta_{\breve{I}_{j}}(x), v_{\breve{I}_{j}}(x)\right)$, where $\hat{J}=1, \cdots, n$. Then, the aggregated value is also SFN by using the ST - SFOWG operator, and is given by

$$
\begin{aligned}
S T & -\operatorname{SFOWG}\left(\breve{I}_{1}, \cdots, \breve{I_{n}}\right) \\
& =\left(\begin{array}{c}
\prod_{\hat{J}=1}^{n}\left(\sin \left(\frac{\pi}{2}\left(\mu_{\sigma(\hat{J})}(x)\right)\right)\right)^{\omega_{\hat{J}}}, \\
\sqrt{1-\prod_{\hat{J}=1}^{n}\left(2 \sin ^{2}\left(\frac{\pi}{4} \eta_{\sigma(\hat{J})}^{2}(x)\right)\right)^{\omega_{\hat{J}}}}, \\
\sqrt{1-\prod_{\hat{J}=1}^{n}\left(2 \sin ^{2}\left(\frac{\pi}{4} v_{\sigma(\hat{J})}^{2}(x)\right)\right)^{\omega_{\hat{J}}}}
\end{array}\right) .
\end{aligned}
$$

Proof Similar to Theorem (3)

Definition 13 A sine trigonometric spherical fuzzy hybrid geometric operator $(S T-S F H G)$ is a mapping $S T$ $S F H G: \Psi^{n} \rightarrow \Psi$, such that the associate vectors are $\xi=\left(\xi_{1}, \xi_{2}, \cdots, \xi_{n}\right)^{T}$, which fulfilled the condition $\xi_{\hat{J}}>0$ and $\sum_{\hat{J}=1}^{n} \xi_{\hat{J}}=1$.

$$
S T-S F H G=\left(\sin \ddot{I}_{\sigma(1)}\right)^{\xi_{1}} \otimes \cdots \otimes\left(\sin \ddot{I}_{\sigma(n)}\right)^{\xi_{n}} .
$$

Where $\sigma$ is the permutation of $(1, \cdots, n)$ as $\breve{I}_{\sigma(\hat{J}-1)} \geq \breve{I}_{\sigma(\hat{J})}$ for any $\hat{J}$ and $\ddot{I}_{\hat{J}}=n \omega_{\hat{J}} \breve{I}_{\hat{J}}$

Theorem 8 Let a family of SFNs are $\breve{I}_{\hat{J}}=$ $\left(\mu_{\breve{I}_{\hat{j}}}(x), \eta_{\breve{I}_{\hat{j}}}(x), v_{\breve{I}_{\hat{j}}}(x)\right)$, where $\hat{J}=1, \cdots, n$. Then, by 
utilizing the operator i.e., ST-SFHG, the aggregated value is also SFN and is given by

$$
\begin{aligned}
S T & -\operatorname{SFHG}\left(\breve{I}_{1}, \cdots, \breve{I_{n}}\right) \\
& =\left(\begin{array}{c}
\prod_{\hat{J}=1}^{n}\left(\sin \left(\frac{\pi}{2}\left(\widetilde{\mu}_{\sigma(\hat{J})}(x)\right)\right)\right)^{\omega_{\hat{J}}}, \\
\sqrt{1-\prod_{\hat{J}=1}^{n}\left(2 \sin ^{2}\left(\frac{\pi}{4} \widetilde{\eta}_{\sigma(\hat{J})}^{2}(x)\right)\right)^{\omega_{\hat{J}}}}, \\
\sqrt{1-\prod_{\hat{J}=1}^{n}\left(2 \sin ^{2}\left(\frac{\pi}{4} \widetilde{v}_{\sigma(\hat{J})}^{2}(x)\right)\right)^{\omega_{\hat{J}}}}
\end{array}\right) .
\end{aligned}
$$

Proof Proof is same to Theorem: (3), so proof is ignore here.

As similar to $S T-S F W A, S T-S F O W A, \quad S T-$ $S F H A, S T-S F W G, S T-S F O W G$ and $S T-S F H G$ operators satisfy the properties such as Idempotency, Boundedness, Monotonicity.

\section{Fundamental properties of the proposed aggregation operators}

In this section of the paper, we discuss many relation between the proposed AOs and also studied their fundamental properties as given,

Theorem 9 For any two SFNs i.e., $\breve{I_{1}}$ and $\breve{I_{2}}$, we have $\sin \breve{I_{1}} \oplus \sin \breve{I_{2}} \geq \sin \breve{I_{1}} \otimes \sin \breve{I_{2}}$

Proof Let the two SFNs are $\breve{I_{1}}=\left(\mu_{\breve{I_{1}}}(x), \eta_{\breve{I_{1}}}(x), v_{\breve{I_{1}}}(x)\right)$ and $\breve{I_{2}}=\left(\mu_{\breve{I_{2}}}(x), \eta_{\breve{I_{2}}}(x), v_{\breve{I_{2}}}(x)\right)$. Then, by using definition (6), (7), we get

$$
\begin{aligned}
& \sin \breve{I_{1}} \oplus \sin \breve{I_{2}}
\end{aligned}
$$

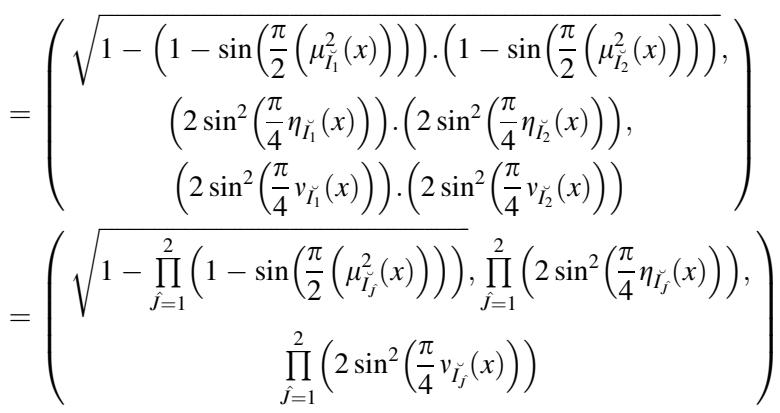

and also $\sin \breve{I_{1}} \otimes \sin \breve{I_{2}}$

$$
\begin{aligned}
& =\left(\begin{array}{c}
\left(\sin \left(\frac{\pi}{2}\left(\mu_{\breve{I_{1}}}(x)\right)\right)\right) \cdot\left(\sin \left(\frac{\pi}{2}\left(\mu_{\breve{I_{2}}}(x)\right)\right)\right), \\
\sqrt{1-\left(2 \sin ^{2}\left(\frac{\pi}{4} \eta_{\breve{I_{1}}}^{2}(x)\right)\right) \cdot\left(2 \sin ^{2}\left(\frac{\pi}{4} \eta_{\breve{I_{2}}}^{2}(x)\right)\right)}, \\
\sqrt{1-\left(2 \sin ^{2}\left(\frac{\pi}{4} v_{\breve{I_{1}}}^{2}(x)\right)\right) \cdot\left(2 \sin ^{2}\left(\frac{\pi}{4} v_{\breve{I_{2}}}^{2}(x)\right)\right)}
\end{array}\right) \\
& =\left(\frac{\prod_{\hat{J}=1}^{2}\left(\sin \left(\frac{\pi}{2}\left(\mu_{\breve{I_{\hat{J}}}}(x)\right)\right)\right), \sqrt{1-\prod_{\hat{J}=1}^{2}\left(2 \sin ^{2}\left(\frac{\pi}{4} \eta_{\breve{J}_{\hat{J}}}^{2}(x)\right)\right)},}{\sqrt{1-\prod_{\hat{J}=1}^{2}\left(2 \sin ^{2}\left(\frac{\pi}{4} v_{I_{\hat{J}}}^{2}(x)\right)\right)}}\right)
\end{aligned}
$$

since for any two non-negative real number $a$ and $b$, their arithmetic mean is greater than or equal to their geometric mean therefore, $\frac{a+b}{2} \geq a b$ which follows that $a+b-$ $a b \geq a b$. Thus by taking $a=\sin \left(\frac{\pi}{2}\left(\mu_{\breve{I_{1}}}(x)\right)\right)$ and $b=$ $\sin \left(\frac{\pi}{2}\left(\mu_{\breve{I_{2}}}(x)\right)\right)$, we have $1-\left(1-\sin \frac{\pi}{2}\left(\mu_{\breve{I_{1}}}(x)\right)\right)$. $\left(1-\sin \frac{\pi}{2}\left(\mu_{2}(x)\right)\right) \geq\left(\sin \frac{\pi}{2}\left(\mu_{\breve{I_{1}}}(x)\right)\right) \cdot\left(\sin \frac{\pi}{2}\left(\mu_{\breve{I_{2}}}(x)\right)\right)$ which further gives that

$$
1-\prod_{\hat{J}=1}^{2}\left(1-\sin \frac{\pi}{2}\left(\mu_{\breve{I_{\hat{J}}}}(x)\right)\right) \geq \prod_{\hat{J}=1}^{2}\left(\sin \frac{\pi}{2}\left(\mu_{\breve{I_{1}}}(x)\right)\right) \text {. }
$$

Similarly, we have obtained the other two as

$$
\prod_{\hat{J}=1}^{2}\left(2 \sin ^{2}\left(\frac{\pi}{4} \eta_{\breve{I_{\hat{J}}}}(x)\right)\right) \leq 1-\prod_{\hat{J}=1}^{2}\left(2 \sin ^{2}\left(\frac{\pi}{4} \eta_{\breve{I_{\hat{J}}}}(x)\right)\right)
$$

and

$$
\prod_{\hat{J}=1}^{2}\left(2 \sin ^{2}\left(\frac{\pi}{4} v_{\breve{I_{f}}}(x)\right)\right) \leq 1-\prod_{\hat{J}=1}^{2}\left(2 \sin ^{2}\left(\frac{\pi}{4} v_{\breve{I}_{\hat{J}}}(x)\right)\right) .
$$

Hence, using Definition (7), we get $\sin \breve{I_{1}} \oplus \sin \breve{I_{2}} \geq \sin \breve{I_{1}} \otimes \sin \breve{I_{2}}$

Theorem 10 For any SFNs i.e., $\breve{I}$ and positive real number $\dot{\tau}>0, \quad \dot{\tau} \sin \breve{I} \quad \geq(\sin \breve{I})^{i} \Longleftrightarrow \dot{\tau} \geq 1 \quad$ and $\quad \dot{\tau} \sin \breve{I}$ $\leq(\sin \breve{I})^{i} \Longleftrightarrow 0<\dot{\tau} \leq 1$.

Proof Proof is same to Theorem (9).

Lemma 1 For $a_{\hat{J}} \geq 0$ and $b_{\hat{J}} \geq 0$, then we have $\prod_{\hat{J}=1}^{n} a_{\hat{J}}^{b_{\hat{J}}} \leq \sum_{\hat{J}=1}^{n} b_{\hat{J}} \cdot a_{\hat{J}} \quad$ and the equality holds iff $a_{1}=a_{2}=\cdots=a_{n}$. 
Lemma 2 Let $0 \leq a, b \leq 1$, and $0 \leq x \leq 1$, then $0 \leq a x+b(1-x) \leq 1$.

\section{Lemma 3 Let $0 \leq a, b \leq 1$, \\ $\sqrt{1-\left(1-a^{2}\right)\left(1-b^{2}\right)} \geq a b$.}

Theorem 11 For any SFNs ${\breve{I_{J}}}_{\hat{J}}=\left(\mu_{\breve{I}_{\hat{j}}}(x), \eta_{\breve{I}_{\hat{j}}}(x), v_{\breve{I_{j}}}(x)\right)$, the operators $S T-S F W A$ and $S T-S F W G$ satisfy the inequality

$S T-S F W A\left(\breve{I_{1}}, \cdots, \breve{I_{n}}\right) \geq S T-S F W G\left(\breve{I_{1}}, \cdots, \breve{I_{n}}\right)$.

Where equality holds iff $\breve{I_{1}}=\breve{I_{2}}=\cdots=\breve{I_{n}}$.

Proof For $n$, SFNs $\breve{I_{\hat{J}}}=\left(\mu_{\breve{I}_{\hat{j}}}(x), \eta_{\breve{I}_{\hat{j}}}(x), \nu_{\breve{I}_{\hat{j}}}(x)\right)$ and normalized weight vector $\omega_{\hat{J}}>0$, we have

$$
\begin{aligned}
S T & -S F W A\left(\breve{I_{1}}, \cdots, \breve{I_{n}}\right) \\
& =\left(\begin{array}{c}
\sqrt{1-\prod_{\hat{J}=1}^{n}\left(1-\sin \left(\frac{\pi}{2}\left(\mu_{\breve{I}_{j}}^{2}(x)\right)\right)\right)^{\omega_{\hat{J}}}}, \\
\prod_{\hat{J}=1}^{n}\left(2 \sin ^{2}\left(\frac{\pi}{4} \eta_{\breve{I}_{\hat{J}}}(x)\right)\right)^{\omega_{\hat{J}}}, \\
\prod_{\hat{J}=1}^{n}\left(2 \sin ^{2}\left(\frac{\pi}{4} v_{\breve{I}_{\hat{J}}}(x)\right)\right)^{\omega_{j}}
\end{array}\right)
\end{aligned}
$$

and

$$
\begin{aligned}
S T & -S F W G\left(\breve{I_{1}}, \cdots, \breve{I_{n}}\right) \\
& =\left(\begin{array}{c}
\prod_{\hat{J}=1}^{n}\left(\sin \left(\frac{\pi}{2}\left(\mu_{\breve{I}_{\hat{J}}}(x)\right)\right)\right)^{\omega_{\hat{J}}}, \\
\sqrt{1-\prod_{\hat{J}=1}^{n}\left(2 \sin ^{2}\left(\frac{\pi}{4} \eta_{\breve{I}_{J}}^{2}(x)\right)\right)^{\omega_{\hat{J}}}}, \\
\sqrt{1-\prod_{\hat{J}=1}^{n}\left(2 \sin ^{2}\left(\frac{\pi}{4} v_{\breve{I}_{J}}^{2}(x)\right)\right)^{\omega_{\hat{J}}}}
\end{array}\right)
\end{aligned}
$$

For $\omega_{\hat{J}}>0, \sin \left(\frac{\pi}{2}\left(\mu_{\hat{J}}\right)\right) \in[0,1]$ and by the Lemma (1), we get

$$
\begin{aligned}
1- & \prod_{\hat{J}=1}^{n}\left(1-\sin \left(\frac{\pi}{2}\left(\mu_{\breve{I}_{\hat{j}}}(x)\right)\right)\right)^{\omega_{\hat{J}}} \\
& \geq 1-\sum_{\hat{J}=1}^{n} \omega_{\hat{J}}\left(1-\sin \left(\frac{\pi}{2}\left(\mu_{I_{\hat{J}}}(x)\right)\right)\right) \\
& \geq 1-1+\sum_{\hat{J}=1}^{n} \omega_{\hat{J}}\left(\sin \left(\frac{\pi}{2}\left(\mu_{\breve{I}_{\hat{J}}}(x)\right)\right)\right) \\
& \left.\geq \prod_{\hat{J}=1}^{n}\left(\sin \left(\frac{\pi}{2}\left(\mu_{\breve{I_{j}}}(x)\right)\right)\right)\right)^{\omega_{\hat{J}}}
\end{aligned}
$$

which implies that

$$
\begin{aligned}
& \sqrt{1-\prod_{\hat{J}=1}^{n}\left(1-\sin \left(\frac{\pi}{2}\left(\mu_{\breve{I}_{j}}^{2}(x)\right)\right)\right)^{\omega_{j}}} \\
& \geq \sqrt{\prod_{\hat{J}=1}^{n}\left(\sin \left(\frac{\pi}{2}\left(\mu_{I_{\hat{J}}}^{2}(x)\right)\right)\right)^{\omega_{j}}} .
\end{aligned}
$$

For neutral and negative membership component, we have

$$
\begin{aligned}
& \prod_{\hat{J}=1}^{n}\left(2 \sin ^{2}\left(\frac{\pi}{4} \eta_{\breve{I}_{\hat{J}}}(x)\right)\right)^{\omega_{\hat{J}}} \\
& \leq \sum_{\hat{J}=1}^{n} \omega_{\hat{J}} \cdot\left(2 \sin ^{2}\left(\frac{\pi}{4} \eta_{\breve{I}_{j}}(x)\right)\right) \\
& \leq 1-\sum_{\hat{J}=1}^{n} \omega_{\hat{J}} \cdot\left(2 \sin ^{2}\left(\frac{\pi}{4} \eta_{\breve{I}_{\hat{J}}}(x)\right)\right) \\
& \leq 1-\prod_{\hat{J}=1}^{n}\left(2 \sin ^{2}\left(\frac{\pi}{4} \eta_{I_{\tilde{J}}}(x)\right)\right)^{\omega_{\hat{J}}}
\end{aligned}
$$

which implies that

$$
\begin{aligned}
& \prod_{\hat{J}=1}^{n}\left(2 \sin ^{2}\left(\frac{\pi}{4} \eta_{\breve{I}_{\hat{J}}}(x)\right)\right)^{\omega_{\hat{J}}} \\
& \quad \leq 1-\prod_{\hat{J}=1}^{n}\left(2 \sin ^{2}\left(\frac{\pi}{4} \eta_{\check{I}_{\hat{J}}}(x)\right)\right)^{\omega_{\hat{J}}} .
\end{aligned}
$$

similar the negative grade, as

$$
\begin{aligned}
& \prod_{\hat{J}=1}^{n}\left(2 \sin ^{2}\left(\frac{\pi}{4} v_{\breve{I}_{\hat{J}}}(x)\right)\right)^{\omega_{\hat{J}}} \\
& \quad \leq \sum_{\hat{J}=1}^{n} \omega_{\hat{J}} \cdot\left(2 \sin ^{2}\left(\frac{\pi}{4} v_{\breve{I_{j}}}(x)\right)\right) \\
& \leq 1-\sum_{\hat{J}=1}^{n} \omega_{\hat{J}} \cdot\left(2 \sin ^{2}\left(\frac{\pi}{4} v_{\breve{I_{j}}}(x)\right)\right) \\
& \quad \leq 1-\prod_{\hat{J}=1}^{n}\left(2 \sin ^{2}\left(\frac{\pi}{4} v_{\breve{I}_{\hat{J}}}(x)\right)\right)^{\omega_{\hat{J}}}
\end{aligned}
$$

which implies that

$$
\begin{aligned}
& \prod_{\hat{J}=1}^{n}\left(2 \sin ^{2}\left(\frac{\pi}{4} v_{\breve{I_{\hat{J}}}}(x)\right)\right)^{\omega_{\hat{J}}} \\
& \quad \leq 1-\prod_{\hat{J}=1}^{n}\left(2 \sin ^{2}\left(\frac{\pi}{4} v_{\breve{I_{j}}}(x)\right)\right)^{\omega_{\hat{J}}} .
\end{aligned}
$$

Hence, from all the above Equations, we get $S T-S F W A\left(\breve{I_{1}}, \cdots, \breve{I_{n}}\right) \geq S T-S F W G\left(\breve{I_{1}}, \cdots, \breve{I_{n}}\right)$. 
Theorem 12 Let

$$
\breve{I}_{\hat{J}}=\left(\mu_{\breve{I}_{\hat{J}}}(x), \eta_{\breve{I}_{\hat{J}}}(x), v_{\breve{I}_{\hat{J}}}(x)\right)(\hat{J}=1, \cdots, n)
$$

and $\breve{I}=\left(\mu_{I}(x), \eta_{I}(x), v_{I}(x)\right)$ are SFNs. Then,

$$
\begin{aligned}
& S T-S F W A\left(\breve{I_{1}} \oplus \cdots \oplus \breve{I_{n}}\right) \\
& \quad \geq S T-S F W A\left(\breve{I_{1}} \otimes \cdots \otimes \breve{I_{n}}\right) \\
& S T-S F W G\left(\breve{I_{1}} \oplus \cdots \oplus \breve{I_{n}}\right) \\
& \quad \geq S T-S F W G\left(\breve{I_{1}} \otimes \cdots \otimes \breve{I_{n}}\right)
\end{aligned}
$$

Proof Here, we prove only the first part, while the other parts can be deduced similarly, for this, let $\breve{I}_{\hat{J}}=$ $\left(\mu_{\breve{I}_{\hat{J}}}(x), \eta_{\breve{I}_{\hat{J}}}(x), v_{\breve{I}_{\hat{J}}}(x)\right)$ and $\breve{I}=\left(\mu_{\breve{I}}(x), \eta_{I^{\prime}}(x), v_{I^{\prime}}(x)\right)$, since both $\breve{I_{\hat{J}}}$ and $\breve{I}$ are $S F N s$.

$$
\begin{aligned}
& S T-S F W A\left(\breve{I_{1}} \oplus \cdots \oplus \breve{I_{n}} \oplus \breve{I}\right) \\
& =\left(\begin{array}{c}
\sqrt{1-\prod_{\hat{J}=1}^{n}\left(1-\sin \left(\frac{\pi}{2}\left(1-\left(1-\mu_{\check{I}_{j}}^{2}(x)\right)\left(1-\mu_{\breve{I}}^{2}(x)\right)\right)\right)\right)^{\omega_{j}}}, \\
\prod_{\hat{J}=1}^{n}\left(2 \sin ^{2}\left(\frac{\pi}{4} \eta_{\breve{I}_{j}}(x) \cdot \eta_{\grave{I}}(x)\right)\right)^{\omega_{j}}, \\
\prod_{\hat{J}=1}^{n}\left(2 \sin \left(\frac{\pi}{4} v_{\breve{I}_{j}}(x) \cdot v_{I}(x)\right)\right)^{\omega_{j}}
\end{array}\right)
\end{aligned}
$$

and

$$
\begin{aligned}
S T & -S F W A\left(\breve{I_{1}} \otimes \cdots \otimes \breve{I_{n}} \otimes \breve{I}\right) \\
& =\left(\begin{array}{c}
1-\prod_{\hat{J}=1}^{n}\left(1-\sin \left(\frac{\pi}{2} \mu_{I_{\hat{J}}}(x) \cdot \mu_{I}(x)\right)\right)^{\omega_{\hat{J}}}, \\
\prod_{\hat{J}=1}^{n}\left(2 \sin ^{2}\left(\frac{\pi}{4} \eta_{\breve{I}_{\hat{J}}}(x)\right) \cdot\left(\eta_{\grave{I}}(x)\right)\right)^{\omega_{\hat{J}}}, \\
\prod_{\hat{J}=1}^{n}\left(2 \sin ^{2}\left(\frac{\pi}{4} v_{\breve{I}_{\hat{J}}}(x)\right) \cdot v_{I_{I}}(x)\right)^{\omega_{\hat{J}}}
\end{array}\right)
\end{aligned}
$$

For $\mu_{\breve{I}_{\hat{f}}}(x), \mu_{I}(x) \in[0,1]$ and Lemma (3), we have

$$
\sqrt{1-\left(1-\mu_{\breve{I}_{\hat{J}}}^{2}(x)\right)\left(1-\mu_{\breve{I}}^{2}(x)\right)} \geq \mu_{\breve{I}_{\hat{J}}}(x) \cdot \mu_{I_{I}}(x) .
$$

Since "sine" is an increasing function, we get

$$
\sin \left(\frac{\pi}{2}\left(1-\left(1-\mu_{\check{I}_{J}}(x)\right)\left(1-\mu_{I_{I}}(x)\right)\right)\right) \geq \sin \frac{\pi}{2}\left(\mu_{\breve{I}_{J}}(x) \cdot \mu_{\check{I}}(x)\right),
$$

which gives that

$$
\begin{aligned}
& \Rightarrow \sqrt{1-\sin \left(\frac{\pi}{2}\left(1-\left(1-\mu_{\breve{I}_{j}}^{2}(x)\right)\left(1-\mu_{\breve{I}}^{2}(x)\right)\right)\right)} \\
& \leq \sqrt{1-\sin \frac{\pi}{2}\left(\mu_{\breve{I}_{f}}^{2}(x) \cdot \mu_{\breve{I}}^{2}(x)\right)} \\
& \Rightarrow \sqrt{\prod_{\hat{J}=1}^{n}\left(1-\sin \left(\frac{\pi}{2}\left(1-\left(1-\mu_{\check{I}_{f}}^{2}(x)\right)\left(1-\mu_{\check{I}}^{2}(x)\right)\right)\right)\right)^{\omega_{\hat{J}}}} \\
& \leq \sqrt{\prod_{\hat{J}=1}^{n}\left(1-\sin \frac{\pi}{2}\left(\mu_{I_{\hat{J}}}^{2}(x) \cdot \mu_{\tilde{I}^{2}}^{2}(x)\right)\right)^{\omega_{\hat{J}}}} \\
& \Rightarrow \sqrt{1-\prod_{\hat{J}=1}^{n}\left(1-\sin \left(\frac{\pi}{2}\left(1-\left(1-\mu_{I_{J}}^{2}(x)\right)\left(1-\mu_{\breve{I}}^{2}(x)\right)\right)\right)\right)^{\omega_{j}}} \\
& \geq \sqrt{1-\prod_{\hat{J}=1}^{n}\left(1-\sin \frac{\pi}{2}\left(\mu_{I_{\tilde{f}}}^{2}(x) \cdot \mu_{\breve{I}}^{2}(x)\right)\right)^{\omega_{j}}}
\end{aligned}
$$

Similarly the neutral and negative grade, we get

$$
\begin{aligned}
& \prod_{\hat{J}=1}^{n}\left(2 \sin ^{2}\left(\frac{\pi}{4} \eta_{\breve{I}_{\hat{f}}}(x) \cdot \eta_{I_{I}}(x)\right)\right)^{\omega_{\hat{J}}} \\
& \quad \leq \prod_{\hat{J}=1}^{n}\left(2 \sin ^{2}\left(\frac{\pi}{4} \eta_{\breve{I}_{\hat{J}}}(x)\right) \cdot \eta_{I_{I}}(x)\right)^{\omega_{\hat{J}}} \\
& \prod_{\hat{J}=1}^{n}\left(2 \sin ^{2}\left(\frac{\pi}{4} v_{\breve{I_{\hat{J}}}}(x) \cdot v_{\breve{I}}(x)\right)\right)^{\omega_{\hat{J}}} \\
& \quad \leq \prod_{\hat{J}=1}^{n}\left(2 \sin ^{2}\left(\frac{\pi}{4} v_{\breve{I_{\hat{J}}}}(x)\right) \cdot v_{I^{\prime}}(x)\right)^{\omega_{\hat{J}}}
\end{aligned}
$$

Therefore, from the above equation, we get

$$
\begin{aligned}
& S T-S F W A\left(\breve{I_{1}} \oplus \cdots \oplus \breve{I_{n}}\right) \\
& \quad \geq S T-S F W A\left(\breve{I_{1}} \otimes \cdots \otimes \breve{I_{n}}\right)
\end{aligned}
$$

\section{Decision-making approach}

This section provides a strategy, preceded by an example, to solve the DM problem. For this reason, let it be the $m$ alternative $\left(\hat{\gamma}_{1}, \cdots, \hat{\gamma}_{m}\right)$ that is evaluated by a group of experts under the $n$ different attribute $\left(\breve{G}_{1}, \cdots, \breve{G}_{n}\right)$. That expert test $\hat{\gamma}_{i}$ and $\breve{G}_{j}$ and gives their preferences in terms of SFNs $\alpha_{i j}^{(\kappa)}=\left(\mu_{i j}^{(\kappa)}, \eta_{i j}^{(\kappa)}, v_{i j}^{(\kappa)}\right)$ where $i=1(1) m ; j=1(1) n$; $\kappa=1(1) d$. Then, the value of every alternative $\hat{\gamma}_{i}$ with the $\breve{G}_{j}$ is shown as;

$\hat{\gamma}_{i}=\left[\left(\breve{G}_{1}, \alpha_{i 1}\right),\left(\breve{G}_{2}, \alpha_{i 2}\right), \cdots,\left(\breve{G}_{n}, \alpha_{i n}\right)\right]$,

let $\omega_{j}>0$ be the normalized weights of attribute $\breve{G}_{j}$. The following steps are taken to calculate the best choice. 
Step 1: In terms of decision matrix, summarize the values of each alternative $\hat{D}^{(\kappa)}=\alpha_{i j}^{(\kappa)}$ with $S F S$ information.

Step 2: Aggregate the different preferences $\alpha_{i j}^{(\kappa)}, \kappa=$ $1, \cdots, d$ into $\alpha_{i j}=\left(\mu_{i j}, \eta_{i j}, v_{i j}\right)$ utilizing either $S T-$ $S F W A$ or $S T-S F W G$ by operators.

Step 3: Establish the normalized decision matrix $R=$ $\left(r_{i j}\right)$ from $\hat{D}=\left(\alpha_{i j}\right)$, where $r_{i j}$ is computed as

$r_{i j}=\left\{\begin{array}{c}\left(\mu_{i j}, \eta_{i j}, v_{i j}\right) \text { if benefit type attributes } \\ \left(v_{i j}, \eta_{i j}, \mu_{i j}\right) \text { if cost type attributes }\end{array}\right.$

Step 4: If the weights of the attributes are known as before, then use them. Otherwise, we measure these using the entropy principle. For this, the information entropy of attribute $\breve{G}_{j}$ is given as;

$$
\begin{aligned}
\Xi_{j}= & \frac{1}{(\sqrt{2}-1) m} \sum_{j=1}^{m}\left[\sin \left(\frac{\pi}{4}\left(1+\mu_{i j}-\eta_{i j}-v_{i j}\right)\right)\right. \\
& \left.+\sin \left(\frac{\pi}{4}\left(1-\mu_{i j}+\eta_{i j}+v_{i j}\right)\right)-1\right],
\end{aligned}
$$

where $\frac{1}{(\sqrt{2}-1) m}$ is a constant for assuring $0 \leq \Xi_{j} \leq 1$.

Based on the Equation (31), the weights of the attributes are obtained as $\omega=\left(\omega_{1}, \cdots, \omega_{n}\right)$, where

$$
\omega_{j}=\frac{1-\Xi_{j}}{n-\sum_{j=1}^{n} \Xi_{j}}
$$

Step 5: With weight vector $\omega$ and using the proposed ST-SFOWA or ST-SFOWG aggregation operators, the collective values $r_{i}$ for each alternative $\hat{\gamma}_{i}$ are calculated.

Step 6: Find the score values of $r_{i}(i=1, \ldots, m)$

Step 7: Grade all the possible alternative $\hat{\gamma}_{i}(i=1, \ldots, m)$ and select the most desirable alternative(s).

\section{Illustrative example}

In this section, the results of the established MAGDM approach are reviewed with the example and their results are compared with those of the current MAGDM approaches.

\subsection{Application of the proposed MAGDM method}

\subsubsection{Selection of an authentic lab for the COVID - 19 test.}

COVID-19 is a pandemic disease. "CO" stands for Corona, "VI" stands for Virus, and "D" stands for Disease. Simply, this disease is called "Corona Virus 2019". Some people infected with the COVID-19 virus suffer from respiratory failure, and some recover without needing special treatment. Older people and those with conditions such as cardiovascular disease, diabetes, chronic respiratory disorders and cancer are more likely to experience serious illnesses. In most cases, COVID-19 spreads via the sneezing or coughing of an infected person. It is also important to observe the respiratory protocol, for example, by coughing into a flexed elbow. There are currently definite vaccines or medications available for the treatment of COVID-19 patients. Nevertheless, scientists and pharmacists are working hard to test new therapies through clinical trials.

Different countries are seeking to monitor and reduce the spread of this virus by quarantining people, restricting travel, monitoring and treating patients, performing contact tracing and canceling large-scale gatherings. This pandemic is moving like a waveone that has quickly infected a lot of people in a few months. We may say that COVID-19 is much more than just a health issue. COVID-19 adversely affects the cultural, social and political environments of any nation in the world.

For such a serious situation as COVID-19, it is mandatory for people who have symptoms of this infection to take a medical test. For this reason, an authentic lab was needed. The main aim of this application is to choose an authentic lab from various laboratories for the COVID-19 test by applying the ST-SFWA and ST-SFWG operators.

Assume that the five labs $\hat{\gamma}_{1}, \hat{\gamma}_{2}, \hat{\gamma}_{3}, \hat{\gamma}_{4}$, and $\hat{\gamma}_{5}$ were assessed by three experts $E_{(1)}, E_{(2)}$ and $E_{(3)}$ for funding focused on three attribute, which are given below,

$\breve{G}_{1}$ denoted time limits,

$\breve{G}_{2}$ denoted accurate results,

$\breve{G}_{3}$ denoted location flexibility for the client.

Assume that $\omega=(0.33,0.37,0.30)$ is the experts weight information and assessment of the decision matrices using SFNs in the following Table 1,2 and 3. Then, we proceed to the selection of the appropriate alternative (lib).

Step 1: The evaluation of each expert is summarized in Table 1, 2 and 3.

Step 2. By taking the weight of the experts i.e., $\omega=$ $(0.33,0.37,0.30)^{T}$ and utilize the $S T-S F W A$ operator to achieve the collective data on each alternative of the expert. The result are shown in Table 4.

Step 3: Almost all the three attributes are just to be the benefit types, then normalization are not needed.

Step 4: Since the attributes weight are completely unknown. Thus, by utilizing the data of the Table 2 and idea of the entropy Equ. (31). We obtain the values; 
Table 1 Decision matrix given by expert $E_{(1)}$

\begin{tabular}{llll}
\hline & $\breve{G}_{1}$ & $\breve{G}_{2}$ & $\breve{G}_{3}$ \\
\hline$\hat{\gamma}_{1}$ & $(0.29,0.54,0.61)$ & $(0.44,0.59,0.56)$ & $(0.60,0.31,0.33)$ \\
$\hat{\gamma}_{2}$ & $(0.54,0.44,0.63)$ & $(0.61,0.48,0.54)$ & $(0.55,0.34,0.36)$ \\
$\hat{\gamma}_{3}$ & $(0.27,0.65,0.68)$ & $(0.73,0.43,0.42)$ & $(0.51,0.55,0.27)$ \\
$\hat{\gamma}_{4}$ & $(0.30,0.22,0.63)$ & $(0.60,0.47,0.63)$ & $(0.46,0.47,0.37)$ \\
$\hat{\gamma}_{5}$ & $(0.54,0.55,0.49)$ & $(0.71,0.54,0.42)$ & $(0.41,0.53,0.46)$ \\
\hline
\end{tabular}

Table 2 Decision matrix given by expert $E_{(2)}$

\begin{tabular}{llll}
\hline & $\breve{G}_{1}$ & $\breve{G}_{2}$ & $\breve{G}_{3}$ \\
\hline$\hat{\gamma}_{1}$ & $(0.42,0.34,0.68)$ & $(0.56,0.47,0.37)$ & $(0.74,0.26,0.30)$ \\
$\hat{\gamma}_{2}$ & $(0.78,0.42,0.44)$ & $(0.67,0.24,0.49)$ & $(0.81,0.20,0.29)$ \\
$\hat{\gamma}_{3}$ & $(0.59,0.37,0.51)$ & $(0.44,0.62,0.34)$ & $(0.46,0.44,0.53)$ \\
$\hat{\gamma}_{4}$ & $(0.47,0.39,0.54)$ & $(0.49,0.58,0.42)$ & $(0.34,0.66,0.40)$ \\
$\hat{\gamma}_{5}$ & $(0.56,0.36,0.48)$ & $(0.50,0.25,0.55)$ & $(0.52,0.35,0.53)$ \\
\hline
\end{tabular}

Table 3 Decision matrix given by expert $E_{(3)}$

\begin{tabular}{llll}
\hline & $\breve{G}_{1}$ & $\breve{G}_{2}$ & $\breve{G}_{3}$ \\
\hline$\hat{\gamma}_{1}$ & $(0.53,0.27,0.36)$ & $(0.51,0.31,0.48)$ & $(0.55,0.33,0.42)$ \\
$\hat{\gamma}_{2}$ & $(0.61,0.38,0.51)$ & $(0.54,0.47,0.29)$ & $(0.65,0.29,0.55)$ \\
$\hat{\gamma}_{3}$ & $(0.58,0.45,0.27)$ & $(0.59,0.33,0.68)$ & $(0.61,0.42,0.38)$ \\
$\hat{\gamma}_{4}$ & $(0.42,0.39,0.57)$ & $(0.58,0.26,0.52)$ & $(0.81,0.23,0.49)$ \\
$\hat{\gamma}_{5}$ & $(0.26,0.64,0.50)$ & $(0.27,0.59,0.44)$ & $(0.44,0.39,0.57)$ \\
\hline
\end{tabular}

$\Xi_{1}=0.9498, \Xi_{2}=0.9194, \Xi_{3}=0.8750$.

By the help of this, we find the attribute weights $\omega=$ $(0.3461,0.3351,0.3188)^{T}$. In Fig. 1, we show graphically the weight vector of the attributes as;

Step 5: Based on $\omega=(0.3461,0.3351,0.3188)^{T}$ and utilizing the $S T-P F W A$ operator, the collective values of each alternatives are gain as;

$$
\begin{aligned}
& \gamma_{1}=(0.452,0.474,0.383), \\
& \gamma_{2}=(0.552,0.361,0.284), \\
& \gamma_{3}=(0.621,0.243,0.264), \\
& \gamma_{4}=(0.398,0.528,0.424), \\
& \gamma_{5}=(0.628,0.343,0.297) .
\end{aligned}
$$

Step 6: We can get the scores of each alternative by using the Equ. (3);

$$
\begin{aligned}
\overline{S c}\left(\gamma_{1}\right) & =0.543, \overline{S c}\left(\gamma_{2}\right)=0.629, \overline{S c}\left(\gamma_{3}\right) \\
& =0.600, \overline{S c}\left(\gamma_{4}\right)=0.502, \overline{S c}\left(\gamma_{5}\right)=0.674 .
\end{aligned}
$$

Step 7: According to the score values as $\overline{S c}\left(\gamma_{5}\right)>\bar{S} c\left(\gamma_{2}\right)>\bar{S} c\left(\gamma_{3}\right)>\bar{S} c\left(\gamma_{1}\right)>\bar{S} c\left(\gamma_{4}\right)$. Thus, the ranking order is $\hat{\gamma}_{5}>\hat{\gamma}_{2}>\hat{\gamma}_{3}>\hat{\gamma}_{1}>\hat{\gamma}_{4}$. Hence, $\hat{\gamma}_{5}$ is the best alternative.

During Step 5 of the established method, the complete analysis by changing aggregation operators is analyzed and their results are shown in Table 5.

We can, therefore, conclude from all the above-mentioned computational process that the alternative $\hat{\gamma}_{2}$ is really the best option amongst the other options and therefore it is strongly recommended that the appropriate option is $\hat{\gamma}_{2}$.

\subsection{Comparative analysis}

In this subsection, we give some brief discussion on the comparison of the proposed method with some well know related methods.

The comparison of the developed approach with the existing approaches to examine the reliability and effectiveness of the explored method. The established method are compared with the some other methods based on SFS was established by Ashraf et al. (2019a), Spherical fuzzy Dombi aggregation operators and their application in group DM problems, Ashraf et al. (2019b), Spherical fuzzy sets and its representation of Spherical fuzzy t-norms and t-conorms, Kutlu Gundogdu and Kahraman (2018), Spherical fuzzy sets and Spherical fuzzy TOPSIS method, Mahmood et al. (2019), An approach toward DM and medical diagnosis problems using the concept of Spherical
Table 4 Aggregated values of experts using ST-SFWA operator

\begin{tabular}{llll}
\hline & $\breve{G}_{1}$ & $\breve{G}_{2}$ & $\breve{G}_{3}$ \\
\hline$\hat{\gamma}_{1}$ & $(0.561,0.274,0.371)$ & $(0.642,0.242,0.362)$ & $(0.428,0.514,0.283)$ \\
$\hat{\gamma}_{2}$ & $(0.472,0.383,0.481)$ & $(0.361,0.462,0.391)$ & $(0.615,0.326,0.527)$ \\
$\hat{\gamma}_{3}$ & $(0.421,0.281,0.634)$ & $(0.526,0.373,0.473)$ & $(0.426,0.512,0.249)$ \\
$\hat{\gamma}_{4}$ & $(0.631,0.193,0.263)$ & $(0.438,0.254,0.187)$ & $(0.369,0.417,0.532)$ \\
$\hat{\gamma}_{5}$ & $(0.386,0.562,0.362)$ & $(0.297,0.393,0.541)$ & $(0.335,0.318,0.436)$ \\
\hline
\end{tabular}


Fig. 1 Graphical representation of the score values of the Table 6

Table 5 Impact of different AOs and their ranking

Table 6 Ranking order of the proposed operators and existing methods

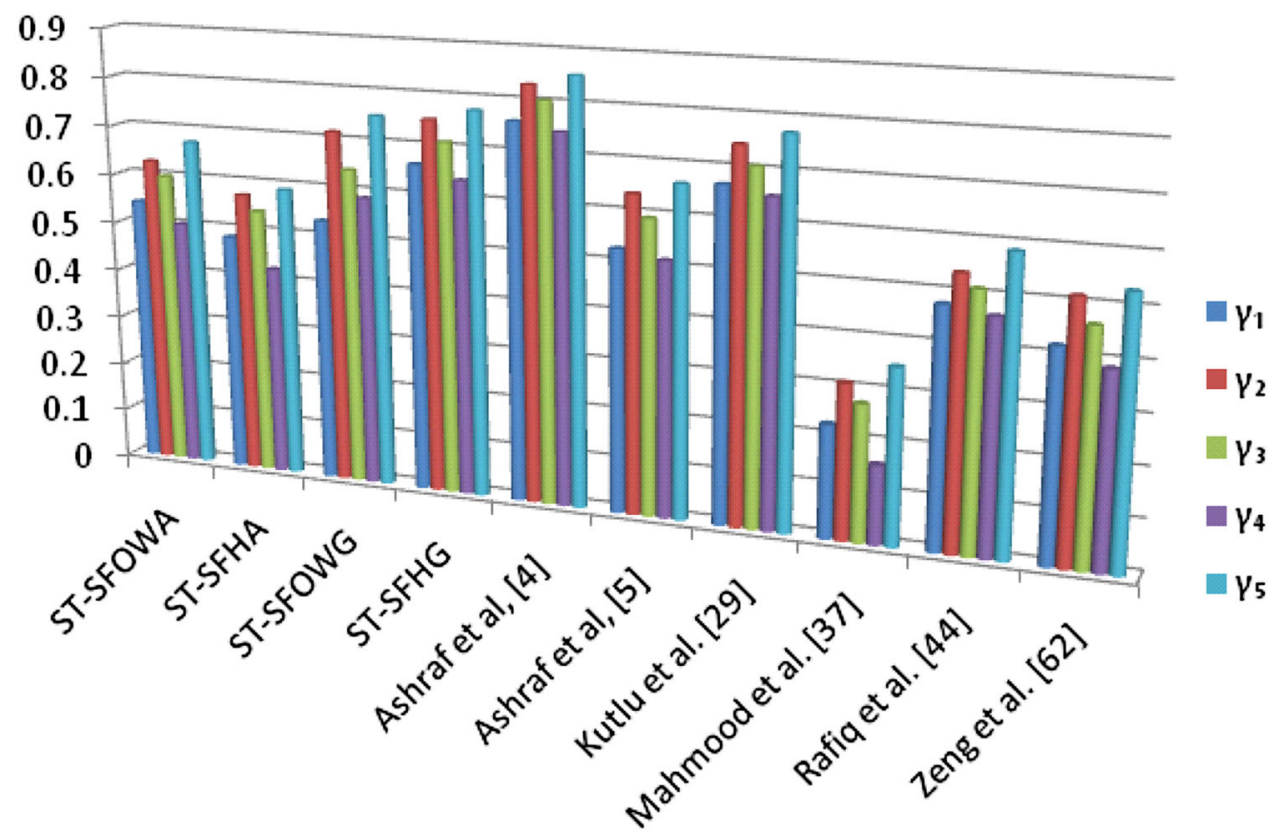

\begin{tabular}{lllllll}
\hline \multirow{2}{*}{ Operators } & \multicolumn{2}{l}{ Score values } & \multicolumn{2}{l}{ Ranking } \\
\cline { 2 - 5 } & $\hat{\gamma}_{1}$ & $\hat{\gamma}_{2}$ & $\hat{\gamma}_{3}$ & $\hat{\gamma}_{4}$ & $\hat{\gamma}_{5}$ & \\
\hline ST-SFOWA & 0.543 & 0.629 & 0.600 & 0.502 & 0.674 & $\hat{\gamma}_{5}>\hat{\gamma}_{2}>\hat{\gamma}_{3}>\hat{\gamma}_{1}>\hat{\gamma}_{4}$ \\
ST-SFHA & 0.484 & 0.573 & 0.542 & 0.425 & 0.591 & $\hat{\gamma}_{5}>\hat{\gamma}_{2}>\hat{\gamma}_{3}>\hat{\gamma}_{1}>\hat{\gamma}_{4}$ \\
ST-SFOWG & 0.534 & 0.716 & 0.642 & 0.587 & 0.753 & $\hat{\gamma}_{5}>\hat{\gamma}_{2}>\hat{\gamma}_{3}>\hat{\gamma}_{4}>\hat{\gamma}_{1}$ \\
ST-SFHG & 0.663 & 0.753 & 0.711 & 0.638 & 0.776 & $\hat{\gamma}_{5}>\hat{\gamma}_{2}>\hat{\gamma}_{3}>\hat{\gamma}_{1}>\hat{\gamma}_{4}$ \\
\hline
\end{tabular}

\begin{tabular}{lllllllll}
\hline \multirow{2}{*}{ Methods } & \multicolumn{3}{l}{ Score values } & \multicolumn{3}{l}{ Ranking } \\
\cline { 2 - 6 } & $\hat{\gamma}_{1}$ & $\hat{\gamma}_{2}$ & $\hat{\gamma}_{3}$ & $\hat{\gamma}_{4}$ & $\hat{\gamma}_{5}$ & \\
\hline ST-SFOWA & 0.543 & 0.629 & 0.600 & 0.502 & 0.674 & $\hat{\gamma}_{5}>\hat{\gamma}_{2}>\hat{\gamma}_{3}>\hat{\gamma}_{1}>\hat{\gamma}_{4}$ \\
ST-SFHA & 0.484 & 0.573 & 0.542 & 0.425 & 0.591 & $\hat{\gamma}_{5}>\hat{\gamma}_{2}>\hat{\gamma}_{3}>\hat{\gamma}_{1}>\hat{\gamma}_{4}$ \\
ST-SFOWG & 0.534 & 0.716 & 0.642 & 0.587 & 0.753 & $\hat{\gamma}_{5}>\hat{\gamma}_{2}>\hat{\gamma}_{3}>\hat{\gamma}_{4}>\hat{\gamma}_{1}$ \\
ST-SFHG & 0.663 & 0.753 & 0.711 & 0.638 & 0.776 & $\hat{\gamma}_{5}>\hat{\gamma}_{2}>\hat{\gamma}_{3}>\hat{\gamma}_{1}>\hat{\gamma}_{4}$ \\
Ashraf et al. (2019a) & 0.761 & 0.832 & 0.804 & 0.746 & 0.855 & $\hat{\gamma}_{5}>\hat{\gamma}_{2}>\hat{\gamma}_{3}>\hat{\gamma}_{1}>\hat{\gamma}_{4}$ \\
Ashraf et al. (2019b) & 0.529 & 0.638 & 0.593 & 0.514 & 0.664 & $\hat{\gamma}_{5}>\hat{\gamma}_{2}>\hat{\gamma}_{3}>\hat{\gamma}_{1}>\hat{\gamma}_{4}$ \\
Kutlu Gundogdu and Kahraman (2018) & 0.669 & 0.746 & 0.707 & 0.653 & 0.773 & $\hat{\gamma}_{5}>\hat{\gamma}_{2}>\hat{\gamma}_{3}>\hat{\gamma}_{1}>\hat{\gamma}_{4}$ \\
Mahmood et al. (2019) & 0.227 & 0.311 & 0.274 & 0.158 & 0.352 & $\hat{\gamma}_{5}>\hat{\gamma}_{2}>\hat{\gamma}_{3}>\hat{\gamma}_{1}>\hat{\gamma}_{4}$ \\
Rafiq et al. (2019) & 0.478 & 0.539 & 0.512 & 0.464 & 0.587 & $\hat{\gamma}_{5}>\hat{\gamma}_{2}>\hat{\gamma}_{3}>\hat{\gamma}_{1}>\hat{\gamma}_{4}$ \\
Zeng et al. (2019) & 0.421 & 0.515 & 0.465 & 0.388 & 0.532 & $\hat{\gamma}_{5}>\hat{\gamma}_{2}>\hat{\gamma}_{3}>\hat{\gamma}_{1}>\hat{\gamma}_{4}$ \\
\hline
\end{tabular}

fuzzy sets, Rafiq et al. (2019), The cosine similarity measures of Spherical fuzzy sets and their applications in DM, Zeng et al. (2019), Covering-based Spherical fuzzy rough set model hybrid with TOPSIS for multiple attribute DM.
From the outcomes of the proposed operators and the other existing methods, we conclude that ranking lists obtained from both the defined method and the compared methods are slightly different, but the best alternative from 
Fig. 2 Graphical representation of the ranking of Table 6

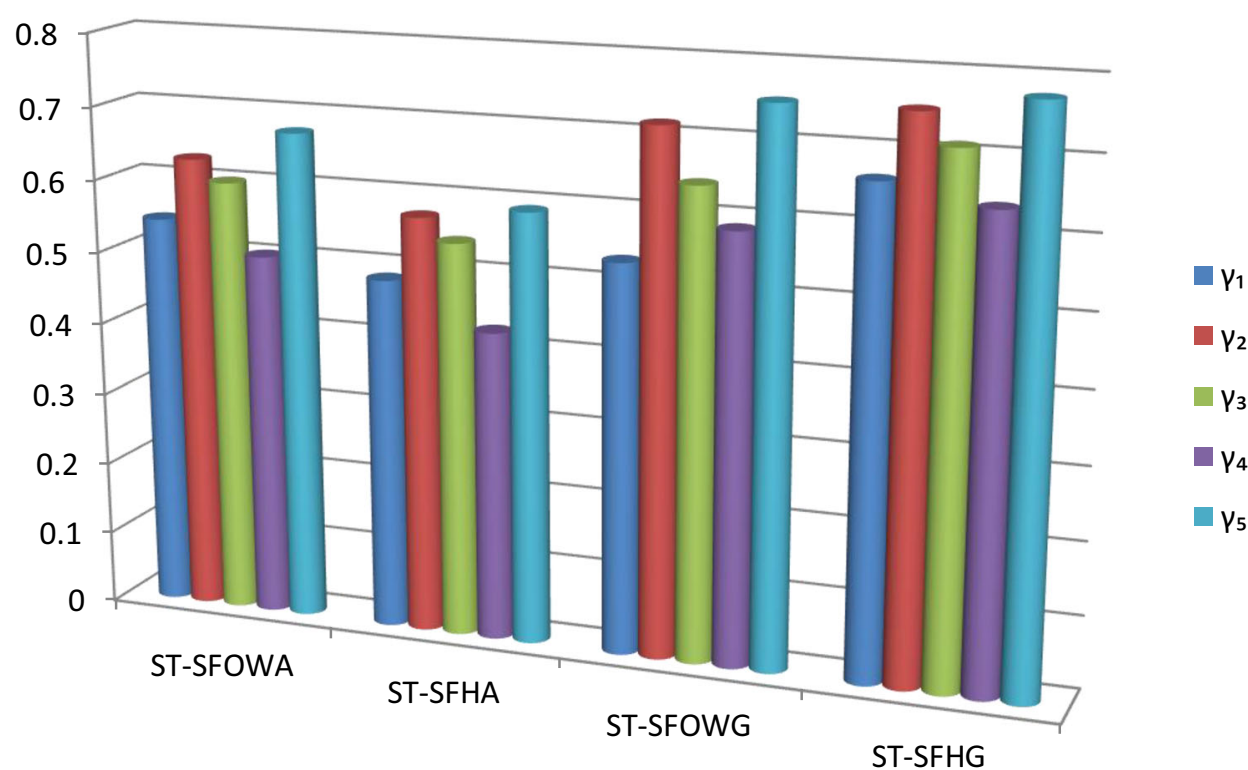

$R^{+}=\left(\zeta_{1}^{+}, \ldots, \zeta_{5}^{+}\right), R^{-}=\left(\zeta_{1}^{-}, \ldots, \zeta_{5}^{-}\right)$.

Where

$\zeta_{j}^{+}=\max \left\{\zeta_{i j} / 1 \leq i \leq 5\right\}$ and $\zeta_{j}^{-}=\min \left\{\zeta_{i j} / 1 \leq i \leq 5\right\}$.

Which are calculated using the score function,

$\bar{S} c(\breve{I})=\mu_{I}(x)+\eta_{I}(x)-v_{I}(x)$.

Step 3 Calculate the distance for each alternative to $R^{+}$ and $R^{-}$using the proposed distance measures with attribute weight vector $\omega=(0.3461,0.3351$, 0.3188).i.e.,

$d_{i}^{+}=\sqrt{\sum_{j=1}^{m} w_{j}\left(\zeta_{j}^{+}-\zeta_{i j}\right)^{2}}$.

And

$d_{i}^{-}=\sqrt{\sum_{j=1}^{m} w_{j}\left(\zeta_{j}^{-}-\zeta_{i j}\right)^{2}}$.

Step 4 Calculate the closeness coefficients to the ideal solution by each alternative by applying the Equation,

$c c_{i}=d_{i}^{-} /\left(d_{i}^{-}+d_{i}^{+}\right)(i=1, . ., 5)$.

The overall closeness coefficients are obtained.

Step 5 Ranking the alternatives using the score function of SFNs and select the best one. We have the ranking result as

$\hat{\gamma}_{5}>\hat{\gamma}_{2}>\hat{\gamma}_{3}>\hat{\gamma}_{1}>\hat{\gamma}_{4}$

All the calculation results and the alternatives ranking is given in Table 7. According to the calculations of overall coefficients, the best one with largest closeness coefficient is $\hat{\gamma}_{5}$. 
Table 7 Calculation Results and Ranking of the Alternatives

\begin{tabular}{|c|c|c|c|c|}
\hline Alternatives & $\begin{array}{l}\text { Distance for alternative to PIS } \\
\left(d_{i}^{+}\right)\end{array}$ & $\begin{array}{l}\text { Distance for alternative to NIS } \\
\left(d_{i}^{-}\right)\end{array}$ & $\begin{array}{l}\text { Closeness coefficients to the ideal solution of } \\
\text { alternative }\left(c c_{i}\right)\end{array}$ & Rank \\
\hline$\hat{\gamma}_{1}$ & 0.172 & 0.111 & 0.392 & 4 \\
\hline$\hat{\gamma}_{2}$ & 0.201 & 0.154 & 0.434 & 2 \\
\hline$\hat{\gamma}_{3}$ & 0.213 & 0.149 & 0.412 & 3 \\
\hline$\hat{\gamma}_{4}$ & 0.372 & 0.131 & 0.261 & 5 \\
\hline$\hat{\gamma}_{5}$ & 0.156 & 0.163 & 0.511 & 1 \\
\hline
\end{tabular}

Hence, by TOPSIS method, it is again verified that $\hat{\gamma}_{5}$ is the most suitable robot to be selected by the manufacturing unit.

\subsubsection{By VIKOR ethod}

Here, we solve the numerical problem given in Section 7 by VIKOR method. The aggregated values of all the individual experts evaluation information based on STSFWA operator is given in Table 4. For this purpose using $\omega=(0.3461,0.3351,0.3188)^{T}$ as the attribute weight vector, we will apply VIKOR method on the information given in Table 4.

Now, to solve the problem using the VIKOR method, the following steps are utilized.

Step 1 Normalize the decision matrix given in Table 4. Here is no need of normalization as all the measure values are of same type, i.e., benefit type.

Step 2 Identifying the PIS $R^{+}$and NIS $R^{-}$. The PIS $R^{+}$and NIS $R^{-}$are defined as follows:

$R^{+}=\left(\zeta_{1}^{+}, \ldots, \zeta_{5}^{+}\right), R^{-}=\left(\zeta_{1}^{-}, \ldots, \zeta_{5}^{-}\right)$.

Where

$\zeta_{j}^{+}=\max \left\{\zeta_{i j} / 1 \leq i \leq 5\right\}$ and $\zeta_{j}^{-}=\min \left\{\zeta_{i j} / 1 \leq i \leq 5\right\}$.

Which are calculated using the score function $\bar{S} c(\breve{I})=\mu_{I}(x)+\eta_{I}(x)-v_{I}(x)$.

Table 8 Ranking of the Alternatives

\begin{tabular}{lllll}
\hline Alternatives & $S_{i}$ & $R_{i}$ & $Q_{i}$ & Rank \\
\hline$\hat{\gamma}_{1}$ & 0.731 & 0.253 & 0.798 & 4 \\
$\hat{\gamma}_{2}$ & 0.637 & 0.163 & 0.431 & 2 \\
$\hat{\gamma}_{3}$ & 0.698 & 0.234 & 0.728 & 3 \\
$\hat{\gamma}_{4}$ & 0.912 & 0.320 & 0.891 & 5 \\
$\hat{\gamma}_{5}$ & 0.401 & 0.172 & 0.00 & 1 \\
\hline
\end{tabular}

Step 3 Calculate the values $S_{i}, R_{i}$ and $Q_{i}$ can be obtained using equations,

$$
\begin{aligned}
S_{i} & =\sum_{j=1}^{m} \frac{w_{j} d\left(\zeta_{i j}, \zeta_{j}^{+}\right)}{d\left(\zeta_{j}^{+}, \zeta_{j}^{-}\right)} . \\
R_{i} & =\max _{i \leq j \leq m} \frac{w_{j} d\left(\zeta_{i j}, \zeta_{j}^{+}\right)}{d\left(\zeta_{j}^{+}, \zeta_{j}^{-}\right)} .
\end{aligned}
$$

And

$Q_{i}=\frac{v\left(S_{i}-S^{*}\right)}{\left(S^{-}-S^{*}\right)}+\frac{(1-v)\left(R_{i}-R^{*}\right)}{\left(R^{-}-R^{*}\right)}$.

Assume $v=0.5$, then the calculated results are shown in the Table 8. Also,

$S^{*}=0.42, S^{-}=0.76, R^{*}=0.33, R^{-}=0.421$.

Step 4 Rank the alternatives by sorting each $S_{i}, R_{i}$, and $Q_{i}$ values in an decreasing order. The values of $Q_{i}$ are ranked as

$$
Q_{4}>Q_{1}>Q_{3}>Q_{2}>Q_{5} \text {. }
$$

Step 5 Propose a compromise solution, from the ranking results, it can be seen that $\hat{\gamma}_{5}$, which is ranked the best by measure $Q_{5}$, is the compromise solution. Thus, both the methods (TOPSIS, VIKOR) have been successfully applied for the verification of the results given by the proposed ST-SFWA aggregation operators for the bset alternative selection. Alternative $\hat{\gamma}_{5}$ is the highest ranked. Hence verified that $\hat{\gamma}_{5}$ is the best alternative among all.

\section{Conclusion}

A research relating to aggregation operators was investigated in this study by establishing some new sine trigonometric operation laws for SFSs. During decisionmaking problems, the well-defined operation laws play a major role. On the other hand, the sine trigonometric function has both the characteristics of the periodicity and 
the symmetrical nature of the origin and, therefore, the most likely to satisfy the experts preference over a multitime period. Therefore, we describe some sine trigonometric operation laws for SFNs and study their properties to take these advantages and make a decision smoother and more important. We have defined various average and geometric AOs on the basis of these operators to club decision makers preference. The different elementary relations between the aggregation operators are discussed and explained in detail. We developed a new MAGDM approach for group DM problems in which goals are classify in terms of SFNs to enforce the proposed laws on decision-making problems. Furthermore, we compute the weight of the attribute by combining the subjective and the objective data in terms of measure. The functionality of the proposed method is applied on an example of selection of an authentic COVID-19 laboratory, and superiority and feasibility of the approach are investigated in detail. A comparative study is often carried out with current works to verify its performance.

In the future, we will use the framework built on new multiple attribute assessment models to tackle fuzziness and ambiguity in a variety of DM parameters, such as design choices, building options, site selection, DM problems, monarch butterfly optimization (MBO) (Feng et al. 2018; Wang et al. 2019d; Feng et al. 2019, 2020), earthworm optimization algorithm (EWA) (Wang et al. 2018a), elephant herding optimization (EHO) (Law et al. 2004; Wang et al. 2016; Li et al. 2020), moth search (MS) algorithm (Wang 2018b), Slime mould algorithm (SMA), and Harris hawks optimization (HHO).

Acknowledgements The authors would like to thank the Deanship of Scientific Research at Umm Al-Qura University for supporting this work by grant number 19-SCI-101-0056.

\section{Compliance with ethical standards}

Conflict of interest The authors declare that they have no conflicts of interest.

\section{References}

Atanassov KT (1986) Intuitionistic fuzzy sets. Fuzzy Sets and System. 20:87-96

Ashraf, S., Mehmood, T., Abdullah, S. and Khan, Q., (2018). Picture fuzzy linguistic sets and their applications for multiattribute group. The Nucleus, 55(2), pp. 66-73

Ashraf, S, Abdullah, S, Mahmood T, Ghani, F. and Mahmood T, (2019a). Spherical fuzzy sets and their applications in multiattribute decision making problems. Journal of Intelligent \& Fuzzy Systems, 36(3), pp. 2829-2844.

Ashraf S, Abdullah S and Mahmood T, (2019b). Spherical fuzzy Dombi aggregation operators and their application in group decision making problems.Journal of Ambient Intelligence and Humanized Computing, pp. 1-19.
Ashraf, S., Abdullah, S., Aslam, M., Qiyas, M. and Kutbi, M.A., (2019c). Spherical fuzzy sets and its representation of spherical fuzzy t-norms and t-conorms. Journal of Intelligent \& Fuzzy Systems, 36(6), pp. 6089-6102

Ashraf, S., Mahmood, T., Abdullah, S. and Khan, Q., (2019d). Different approaches to multi-criteria group decision making problems for picture fuzzy environment. Bulletin of the Brazilian Mathematical Society, New Series, 50(2), pp. 373-397

Akram, M., Bashir, A. and Garg, H., (2020). Decision-making model under complex picture fuzzy Hamacher aggregation operators. Computational and Applied Mathematics, 39(3), pp. 1-38

Cuong BC, Kreinovich V (2013) Picture fuzzy sets-a new concept for computational intelligence problems. In: 2013 third world congress on information and communication technologies (WICT 2013). IEEE, pp 1-6

Cuong BC, Van Hai P (2015) Some fuzzy logic operators for picture fuzzy sets. In: Knowledge and systems engineering (KSE), 2015 seventh international conference on. IEEE, pp 132-137

Cuong BC, Kreinovitch V, Ngan RT (2016) A classification of representable t-norm operators for picture fuzzy sets. In: Knowledge and systems engineering (KSE), (2016). Eighth international conference on. IEEE, pp 19-24

Chen, S.M. and Chang, C.H., (2016a). Fuzzy multiattribute decision making based on transformation techniques of intuitionistic fuzzy values and intuitionistic fuzzy geometric averaging operators. Information Sciences, 352, pp. 133-149

Chen, S.M., Cheng, S.H. and Lan, T.C., (2016b). Multicriteria decision making based on the TOPSIS method and similarity measures between intuitionistic fuzzy values. Information Sciences, 367, pp. 279-295

Chen, S.M., Cheng, S.H. and Lan, T.C., (2016c). A novel similarity measure between intuitionistic fuzzy sets based on the centroid points of transformed fuzzy numbers with applications to pattern recognition. Information Sciences, 343, pp. 15-40

Chen, S.M., Cheng, S.H. and Chiou, C.H., (2016d). Fuzzy multiattribute group decision making based on intuitionistic fuzzy sets and evidential reasoning methodology. Information Fusion, 27, pp. $215-227$

De, S.K., Biswas, R. and Roy, A.R., (2000). Some operations on intuitionistic fuzzy sets. Fuzzy sets and Systems, 114(3), pp. $477-484$

Feng, Y., Wang, G.G., Li, W. and Li, N., (2018). Multi-strategy monarch butterfly optimization algorithm for discounted $0-1$ knapsack problem. Neural Computing and Applications, 30(10), pp. 3019-3036

Feng, Y., Yu, X. and Wang, G.G., (2019). A Novel Monarch Butterfly Optimization with Global Position Updating Operator for LargeScale 0-1 Knapsack Problems. Mathematics, 7(11), p. 1056

Feng, Y., Deb, S., Wang, G.G. and Alavi, A.H., (2020). Monarch butterfly optimization: a comprehensive review. Expert Systems with Applications, p. 114418.

Garg, H., (2016a). Generalized intuitionistic fuzzy interactive geometric interaction operators using Einstein t-norm and $\mathrm{t}$-conorm and their application to decision making. Computers \& Industrial Engineering, 101, pp. 53-69

Gou, X., Xu, Z. and Lei, Q., (2016). New operational laws and aggregation method of intuitionistic fuzzy information. Journal of Intelligent \& Fuzzy Systems, 30(1), pp. 129-141

Garg, H., (2016b). A new generalized Pythagorean fuzzy information aggregation using Einstein operations and its application to decision making. International Journal of Intelligent Systems, 31(9), pp. 886-920

Garg, H., (2017a). Generalized Pythagorean fuzzy geometric aggregation operators using Einstein t-norm and t-conorm for multicriteria decision-making process. International Journal of Intelligent Systems, 32(6), pp. 597-630 
Garg, H., (2017b). Some picture fuzzy aggregation operators and their applications to multicriteria decision-making. Arabian Journal for Science and Engineering, 42(12), pp. 5275-5290

Garg, H., (2017c). Novel intuitionistic fuzzy decision making method based on an improved operation laws and its application. Engineering Applications of Artificial Intelligence, 60, pp. $164-174$

Gou, X. and Xu, Z., (2017). Exponential operations for intuitionistic fuzzy numbers and interval numbers in multi-attribute decision making. Fuzzy Optimization and decision making, 16(2), pp. 183-204

Garg, H. and Kumar, K., (2018a). An advanced study on the similarity measures of intuitionistic fuzzy sets based on the set pair analysis theory and their application in decision making. Soft Computing, 22(15), pp. 4959-4970

Garg H, Kumar K (2018b) A novel exponential distance and its based TOPSIS method for interval-valued intuitionistic fuzzy sets using connection number of SPA theory. Artif Intell Rev 1-30

Gao, H., (2018a). Pythagorean fuzzy hamacher prioritized aggregation operators in multiple attribute decision making. Journal of Intelligent \& Fuzzy Systems, 35(2), pp. 2229-2245

Gao J, Liang, Z, Shang J and Xu Z, (2018). Continuities, Derivatives, and Differentials of -Rung Orthopair Fuzzy Functions. IEEE Transactions on Fuzzy Systems, 27(8), pp. 1687-1699.

Garg, H., (2019a). Generalized intuitionistic fuzzy entropy-based approach for solving multi-attribute decision-making problems with unknown attribute weights. Proceedings of the National Academy of Sciences, India Section A: Physical Sciences, 89(1), pp. $129-139$

Garg H (2019b) Intuitionistic fuzzy hamacher aggregation operators with entropy weight and their applications to multi-criteria decision-making problems. Iran J Sci Technol Trans Electric Eng 1-17

Hwang, C.M., Yang, M.S. and Hung, W.L., (2018). New similarity measures of intuitionistic fuzzy sets based on the Jaccard index with its application to clustering. International Journal of Intelligent Systems, 33(8), pp. 1672-1688

Jin, Y., Ashraf, S. and Abdullah, S., (2019). Spherical fuzzy logarithmic aggregation operators based on entropy and their application in decision support systems. Entropy, 21(7), p. 628

Jin, H., Ashraf, S., Abdullah, S., Qiyas, M., Bano, M. and Zeng, S., (2019). Linguistic spherical fuzzy aggregation operators and their applications in multi-attribute decision making problems. Mathematics, 7(5), p. 413

Kutlu Gundogdu F, Kahraman C (2018) Spherical fuzzy sets and spherical fuzzy TOPSIS method. J Intell Fuzzy Syst (Preprint) $1-16$

Khan, S., Abdullah, S., Abdullah, L. and Ashraf, S., (2019). Logarithmic Aggregation Operators of Picture Fuzzy Numbers for Multi-Attribute Decision Making Problems. Mathematics, 7(7), p. 608

Khan, S., Abdullah, S. and Ashraf, S., (2019). Picture fuzzy aggregation information based on Einstein operations and their application in decision making. Mathematical Sciences, 13(3), pp. $213-229$

Khalil, A.M., Li, S.G., Garg, H., Li, H. and Ma, S., (2019). New operations on interval-valued picture fuzzy set, interval-valued picture fuzzy soft set and their applications. IEEE Access, 7, pp. $51236-51253$

Law, M.H., Topchy, A.P. and Jain, A.K., (2004). Multiobjective data clustering. In Proceedings of the 2004 IEEE Computer Society Conference on Computer Vision and Pattern Recognition, 2004. CVPR 2004. (Vol. 2, pp. II-II). IEEE.

Liu, P., (2013). Some Hamacher aggregation operators based on the interval-valued intuitionistic fuzzy numbers and their application to group decision making. IEEE Transactions on Fuzzy systems, 22(1), pp. 83-97

Liu, P. and Wang, P., (2018a). Multiple-attribute decision-making based on Archimedean Bonferroni Operators of q-rung orthopair fuzzy numbers. IEEE Transactions on Fuzzy systems, 27(5), pp. $834-848$

Liu, P. and Wang, P., (2018b). Some q-rung orthopair fuzzy aggregation operators and their applications to multiple-attribute decision making. International Journal of Intelligent Systems, 33(2), pp. 259-280

Liu, Z., Wang, S. and Liu, P., (2018). Multiple attribute group decision making based on q-rung orthopair fuzzy Heronian mean operators. International Journal of Intelligent Systems, 33(12), pp. $2341-2363$

Liu, P. and Liu, W., (2019a). Multiple-attribute group decisionmaking based on power Bonferroni operators of linguistic q-rung orthopair fuzzy numbers. International Journal of Intelligent Systems, 34(4), pp. 652-689

Liu, P. and Liu, W., (2019b). Multiple-attribute group decisionmaking method of linguistic q-rung orthopair fuzzy power Muirhead mean operators based on entropy weight. International Journal of Intelligent Systems, 34(8), pp. 1755-1794

Liu, P., Munir, M., Mahmood, T. and Ullah, K., (2019). Some similarity measures for interval-valued picture fuzzy sets and their applications in decision making. Information, 10(12), p. 369

Liu, P. and Wang, Y., (2020). Multiple attribute decision making based on q-rung orthopair fuzzy generalized Maclaurin symmetic mean operators. Information Sciences, 518, pp. 181-210

Lin, M., Huang, C. and Xu, Z., (2020). MULTIMOORA based MCDM model for site selection of car sharing station under picture fuzzy environment. Sustainable cities and society, 53, p. 101873

Li, J., Lei, H., Alavi, A.H. and Wang, G.G., (2020). Elephant herding optimization: variants, hybrids, and applications. Mathematics, 8(9), p. 1415

Mahmood, T., Ullah, K., Khan, Q. and Jan, N., (2019). An approach toward decision-making and medical diagnosis problems using the concept of spherical fuzzy sets. Neural Computing and Applications, 31(11), pp. 7041-7053

Nie, R.X., Tian, Z.P., Wang, J.Q. and Hu, J.H., (2019). Pythagorean fuzzy multiple criteria decision analysis based on Shapley fuzzy measures and partitioned normalized weighted Bonferroni mean operator. International Journal of Intelligent Systems, 34(2), pp. 297-324

Phong PH, Hieu DT, Ngan RT, Them PT (2014) Some compositions of picture fuzzy relations. In: Proceedings of the 7 th national conference on fundamental and applied information technology research (FAIR'7), Thai Nguyen, pp 19-20

Peng, X. and Yang, Y., (2015). Some results for Pythagorean fuzzy sets. International Journal of Intelligent Systems, 30(11), pp. $1133-1160$

Peng, X., Dai, J. and Garg, H., (2018). Exponential operation and aggregation operator for q-rung orthopair fuzzy set and their decision-making method with a new score function. International Journal of Intelligent Systems, 33(11), pp. 2255-2282

Qin, J. and Liu, X., (2014). An approach to intuitionistic fuzzy multiple attribute decision making based on Maclaurin symmetric mean operators. Journal of Intelligent \& Fuzzy Systems, 27(5), pp. 2177-2190

Qiyas, M., Abdullah, S., Ashraf, S. and Abdullah, L., (2019). Linguistic Picture Fuzzy Dombi Aggregation Operators and Their Application in Multiple Attribute Group Decision Making Problem. Mathematics, 7(8), p. 764

Rafiq, M., Ashraf, S., Abdullah, S., Mahmood, T. and Muhammad, S., (2019). The cosine similarity measures of spherical fuzzy sets 
and their applications in decision making. Journal of Intelligent \& Fuzzy Systems, 36(6), pp. 6059-6073

Son, L.H., (2017). Measuring analogousness in picture fuzzy sets: from picture distance measures to picture association measures. Fuzzy Optimization and Decision Making, 16, pp. 359-378

Torra, V., (2010). Hesitant fuzzy sets. International Journal of Intelligent Systems, 25(6), pp. 529-539

Wei, G., (2016). Picture fuzzy cross-entropy for multiple attribute decision making problems. Journal of Business Economics and Management, 17(4), pp. 491-502

Wei, G., (2017). Picture fuzzy aggregation operators and their application to multiple attribute decision making. Journal of Intelligent \& Fuzzy Systems, 33(2), pp. 713-724

Wei, G., Alsaadi, F.E., Hayat, T. and Alsaedi, A., (2018). Projection models for multiple attribute decision making with picture fuzzy information. International Journal of Machine Learning and Cybernetics, 9(4), pp. 713-719

Wang, G.G., Deb, S., Gao, X.Z. and Coelho, L.D.S., (2016). A new metaheuristic optimisation algorithm motivated by elephant herding behaviour. International Journal of Bio-Inspired Computation, 8(6), pp. 394-409

Wang, G.G., Deb, S. and Coelho, L.D.S., (2018a). Earthworm optimisation algorithm: a bio-inspired metaheuristic algorithm for global optimisation problems. International Journal of BioInspired Computation, 12(1), pp. 1-22

Wang, G.G., (2018b). Moth search algorithm: a bio-inspired metaheuristic algorithm for global optimization problems. Memetic Computing, 10(2), pp. 151-164

Wang, L., Zhang, H.Y., Wang, J.Q. and Li, L., (2018c). Picture fuzzy normalized projection-based VIKOR method for the risk evaluation of construction project. Applied Soft Computing, 64, pp. 216-226

Wang, R., Wang, J., Gao, H. and Wei, G., (2019a). Methods for MADM with picture fuzzy muirhead mean operators and their application for evaluating the financial investment risk. Symmetry, 11(1), p. 6

Wei, G., Wei, C., Wang, J., Gao, H. and Wei, Y., (2019b). Some q-rung orthopair fuzzy maclaurin symmetric mean operators and their applications to potential evaluation of emerging technology commercialization. International Journal of Intelligent Systems, $34(1)$, pp. $50-81$
Wang, P., Wang, J., Wei, G. and Wei, C., (2019c). Similarity measures of q-rung orthopair fuzzy sets based on cosine function and their applications. Mathematics, 7(4), p. 340

Wang, G.G., Deb, S. and Cui, Z., (2019d). Monarch butterfly optimization. Neural computing and applications, 31(7), pp. 1995-2014

Wei, G., Zhang, S., Lu, J., Wu, J. and Wei, C., (2019). An Extended Bidirectional Projection Method for Picture Fuzzy MAGDM and Its Application to Safety Assessment of Construction Project. IEEE Access, 7, pp. 166138-166147

$\mathrm{Xu}, \mathrm{Z}$. and Yager, R.R., (2006). Some geometric aggregation operators based on intuitionistic fuzzy sets. International journal of general systems, 35(4), pp. 417-433

$\mathrm{Xu}, \mathrm{Z}$. , (2007). Intuitionistic fuzzy aggregation operators. IEEE Transactions on fuzzy systems, 15(6), pp. 1179-1187

Yager RR (2013) Pythagorean fuzzy subsets. In: 2013 joint IFSA world congress and NAFIPS annual meeting (IFSA/NAFIPS). IEEE, pp 57-61

Yager, R.R., 2016. Generalized orthopair fuzzy sets. IEEE Transactions on Fuzzy Systems, 25(5), pp. 1222-1230

Zadeh LA (1965) Fuzzy sets. Information and control. 8:338-353

Zhang, X. and Xu, Z., (2014). Extension of TOPSIS to multiple criteria decision making with Pythagorean fuzzy sets. International Journal of Intelligent Systems, 29(12), pp. 1061-1078

Zeng, S., Asharf, S., Arif, M. and Abdullah, S., (2019a). Application of exponential jensen picture fuzzy divergence measure in multicriteria group decision making. Mathematics, 7(2), p. 191

Zeng, S., Hussain, A., Mahmood, T., Irfan Ali, M., Ashraf, S. and Munir, M., (2019b). Covering-based spherical fuzzy rough set model hybrid with TOPSIS for multi-attribute decision-making. Symmetry, 11(4), p. 547

Zeng, S., Chen, S.M. and Kuo, L.W., (2019c). Multiattribute decision making based on novel score function of intuitionistic fuzzy values and modified VIKOR method. Information Sciences, 488, pp. $76-92$

Publisher's Note Springer Nature remains neutral with regard to jurisdictional claims in published maps and institutional affiliations. 